Pacific Journal of Mathematics

ESTIMATING NIELSEN NUMBERS ON 


\title{
ESTIMATING NIELSEN NUMBERS ON INFRASOLVMANIFOLDS
}

\author{
Christopher K. MCCORD
}

\begin{abstract}
A well-known lower bound for the number of fixed points of a selfmap $f: X \rightarrow X$ is the Nielsen number $N(f)$. Unfortunately, the Nielsen number is difficult to calculate. The Lefschetz number $L(f)$, on the other hand, is readily computable, but does not give a lower bound for the number of fixed points. In this paper, we investigate conditions on the space $X$ which guarantee either $N(f)=|L(f)|$ or $N(f) \geq|L(f)|$. By considering the Nielsen and Lefschetz coincidence numbers, we show that $N(f) \geq|L(f)|$ for all self-maps on compact infrasolvmanifolds (aspherical manifolds whose fundamental group has a normal solvable subgroup of finite index). Moreover, for infranilmanifolds, there is a Lefschetz number formula which computes $N(f)$.
\end{abstract}

1. Estimating Nielsen numbers. Consider a continuous self-map $f: X \rightarrow X$. Let Fix $(f)$ denote the fixed point set $\{x \in X \mid f(x)=x\}$. One of the fundamental problems of fixed point theory is to estimate (preferably from below) the cardinality of this set. The Nielsen number $N(f)$ provides such an estimate: it is an integer homotopy invariant which provides a lower bound on the number of fixed points of $g$, for all maps $g$ homotopic to $f$. This estimate is sharp for all compact manifolds save surfaces of negative Euler characteristic. Its one drawback is that it is very difficult to compute $N(f)$ from its definition, so that other means must be sought. At least, since the Nielsen number provides a lower bound for the original topological object $|\operatorname{Fix}(f)|$, it would be useful to find lower bounds for $N(f)$. We will refer to the search for lower bounds to $N(f)$ as the problem of estimating $N(f)$; while the search for other algebraic-topological means of finding the exact value of $N(f)$ will be referred to as the problem of computing $N(f)$.

The Lefschetz number $L(f)$ is a (reasonably) computable invariant, but in general, there is no relation between $L(f)$ and either $N(f)$ or $|\mathrm{Fix}(f)|$. One approach to computing the Nielsen number is to find conditions on either the space $X$ or the map $f$ which allow $N(f)$ and $L(f)$ to be related. The Jiang condition, for example, is a condition on the map $f$ which, when satisfied, computes $N(f)$ from $L(f)$ 
and $\operatorname{coker}\left(1-f_{1 *}\right)$. The other approach, searching for conditions on the space $X$, begins with the result of Brooks, Brown, Pak and Taylor [8], that $N(f)=|L(f)|$ for all maps on tori. Anosov [3] and Fadell and Husseini [11] show that the equality holds for all maps on compact nilmanifolds. While counter-examples on the Klein bottle show that equality does not hold for all maps on solvmanifolds, nor on infranilmanifolds, Kwasik and Lee [16] show that $N(f)=L(f)$ for homotopically periodic maps on infranilmanifolds, and it is shown in [18] that $N(f) \geq|L(f)|$ for all maps on solvmanifolds.

Fixed-point theory has a natural extension to coincidences: if $f, g$ : $X_{1} \rightarrow X_{2}$, let $\operatorname{Coin}(f, g)=\left\{x \in X_{1} \mid f(x)=g(x)\right\}$. The Nielsen number generalizes to a Nielsen coincidence number $N(f, g)$, which is a homotopy invariant and a lower bound for the number of coincidences. This estimate is sharp when $X_{1}$ and $X_{2}$ are manifolds with $\operatorname{dim}\left(X_{1}\right)=\operatorname{dim}\left(X_{2}\right) \geq 3$. The Lefschetz coincidence number, on the other hand, is only defined when $X_{1}$ and $X_{2}$ are orientable manifolds of the same dimension. Jezierski [14] and Brooks and Wang [9] show that $N(f, g)=|L(f, g)|$ when $X_{1}=X_{2}$ is an infranilmanifold; in [19], it is shown that $N(f, g) \geq|L(f, g)|$ when $X_{1}$ and $X_{2}$ are compact orientable solvmanifolds of the same dimension, with equality if $X_{2}$ is a nilmanifold.

The original goal of this work was to extend the results of [19] to nonorientable solvmanifolds. This was to be done by lifting the map to the orientable double cover (which is also a solvmanifold) and applying the existing results there. All that was needed was to understand the relation between the Nielsen and Lefschetz numbers for the original maps and the numbers for the lifts. Once this relation was investigated and understood, it became clear that a broader class of manifolds could be studied in this manner. The present work therefore studies compact infrasolvmanifolds, manifolds which admit a finite cover by a compact solvmanifold. While the main results (Theorems 7.4 and 7.9) are stated in terms of coincidence numbers, their specialization to fixed point numbers extends the results of Anosov et al. to the following:

If $M$ is a compact infrasolvmanifold, then $N(f) \geq|L(f)|$ for all $f: M \rightarrow M$. If $M$ is a compact infranilmanifold covered by compact nilmanifold $\widetilde{M}$, there is an expression $\widetilde{L}\left(f, \Gamma_{2}\right)$, involving Lefschetz numbers of lifts of $f$ to $\widetilde{M}$, which computes $N(f)$.

It is worth noting that this Lefschetz formula involves Lefschetz coincidence numbers of lifts, even when the original problem involved only fixed point numbers. 
The next two sections contain a brief recapitulation of the relevant parts of Nielsen coincidence theory. Sections 4 and 5 explore the relation between coincidence numbers of maps and coincidence numbers of lifts of the maps. Section 6 describes the topology and algebra of infrasolvmanifolds, while $\S 7$ combines the results of $\S 5$ with the estimates in [18] and [19] to obtain the estimates for Nielsen numbers on infrasolvmanifolds. The paper concludes with a comparison in $\S 8$ between these results and the Jiang condition, and some open questions in $\S 9$.

2. Coincidence numbers. We now briefly review the basics of Nielsen coincidence theory, as developed by Brooks [4], [7]. We will work in the category of compact connected polyhedra and continuous maps. If $X_{1}, X_{2}$ are polyhedra and $f, g: X_{1} \rightarrow X_{2}$ are maps, let Coin $(f, g)=$ $\left\{x \in X_{1} \mid f(x)=g(x)\right\}$ be the coincidence set of $f$ and $g$. To analyze this set, we begin by partitioning it into coincidence classes $\mathbf{S}(f, g)$ (or just $\mathbf{S}$ when $f$ and $g$ are understood). If $x, y \in$ Coin $(f, g)$, set $x \sim y$ if there exists a path $\omega$ in $X_{1}$ from $x$ to $y$ with $f \circ \omega \simeq g \circ \omega(\operatorname{rel}\{0,1\})$. Clearly, each coincidence class is a union of path components of $\operatorname{Coin}(f, g)$, and so is compact and open in $\operatorname{Coin}(f, g)$. Denote the set of coincidence classes of $\operatorname{Coin}(f, g)$ by $\mathscr{R}(f, g)$.

If $F: f_{0} \simeq f_{1}$ and $G: g_{0} \simeq g_{1}$, then coincidence classes $\mathbf{S}_{0} \in$ $\mathscr{R}\left(f_{0}, g_{0}\right)$ and $\mathbf{S}_{1} \in \mathscr{R}\left(f_{1}, g_{1}\right)$ are $(F, G)$-related if there exist $x_{0} \in$ $\mathbf{S}_{0}, x_{1} \in \mathbf{S}_{1}$ and path $\omega$ in $X_{1}$ such that the paths $\langle F, \omega\rangle$ and $\langle G, \omega\rangle$, defined by $\langle F, \omega\rangle(t)=F_{t}(\omega(t))$, are homotopic in $X_{2}$. A class $\mathbf{S} \in \mathscr{R}(f, g)$ is topologically essential if, for every $F: f \simeq$ $f^{\prime}, G: g \simeq g^{\prime}$, there exists a class $\mathbf{S}^{\prime} \in \mathscr{R}\left(f^{\prime}, g^{\prime}\right)$ which is $(F, G)$ related to $\mathbf{S}$. That is, the class cannot be "homotoped away". We will denote the set of topologically essential coincidence classes by $\mathscr{E}(f, g)$. The Nielsen coincidence number $N(f, g)$ is the number of topologically essential coincidence classes of $f$ and $g$.

The Nielsen coincidence number is, by construction, a homotopy invariant and a lower bound on the number of coincidences of $f^{\prime}$ and $g^{\prime}$ for every $f^{\prime} \simeq f$ and $g^{\prime} \simeq g$. We will refer to a pair of manifolds $M_{1}, M_{2}$ as a Wenken pair if $N(f, g)$ is a sharp lower bound for every pair of homotopy classes. All manifolds $M_{1}, M_{2}$ with $\left(M_{2} \times M_{2}, M_{2} \times M_{2} \backslash \Delta\left(M_{2}\right)\right)$ 2-connected (in particular, if $M_{2}$ is an $n$-manifold with $n \geq 3$ ) are Wenken pairs [6]. However, it cannot be used directly to estimate the number of coincidences of $f$ and $g$, since complete information about $\operatorname{Coin}(f, g)$ (indeed, about 
Coin $\left(f^{\prime}, g^{\prime}\right)$ for all $\left.f^{\prime} \simeq f, g^{\prime} \simeq g\right)$ is required before $N(f, g)$ can be computed. So, to make it a useful tool for estimating $\operatorname{Coin}(f, g)$, indirect methods of computation are required. One approach begins by replacing the concept of topologically essential classes with that of algebraically essential classes.

This is done by introducing a coincidence index. A variety of indices are possible in different settings, but the following will suffice for our purposes. Suppose $M_{1}$ and $M_{2}$ are both compact connected orientable $n$-manifolds, with $\left(M_{1} \times M_{2}, M_{2} \times M_{2} \backslash \Delta\left(M_{2}\right)\right)$ 2-connected. For each coincidence class (in fact, for any set $\mathbf{S} \subseteq$ $\operatorname{Coin}(f, g)$ which is open in Coin $(f, g)$ and compact) a coincidence number $\operatorname{Ind}(f, g, \mathbf{S})$ is defined. If $W, V$ are neighborhoods of $\mathbf{S}$ with $\mathbf{S} \subset W \subset \bar{W} \subset V$ and $\operatorname{Coin}(f, g) \cap V=\mathbf{S}$, then let $(f, g)$. be the composition

$$
\begin{aligned}
& H_{n}\left(M_{1}\right) \rightarrow H_{n}\left(M_{1}, M_{1} \backslash W\right) \\
& \stackrel{\cong}{\cong} H_{n}(V, V \backslash W) \stackrel{\left(f_{*}, g_{*}\right)}{\longrightarrow} H_{n}\left(M_{2} \times M_{2}, M_{2} \times M_{2} \backslash \Delta\left(M_{2}\right)\right) \text {. }
\end{aligned}
$$

If $z_{1} \in H_{n}\left(M_{1}\right)$ is the fundamental class of $M_{1}$ and $U_{2} \in$ $H_{n}\left(M_{2} \times M_{2}, M_{2} \times M_{2} \backslash \Delta\left(M_{2}\right)\right)$ is the Thom class of $M_{2}$, then $\operatorname{Ind}(f, g, \mathbf{S}) \equiv\left\langle U_{2},(f, g)_{\bullet}\left(z_{1}\right)\right\rangle$. This is independent of $V$ and $W$, and has the following properties [23]:

1. Coincidence: if $\operatorname{Ind}(f, g, \mathbf{S}) \neq 0$, then $\mathbf{S} \neq \varnothing$.

2. Homotopy: if $F: f_{0} \simeq f_{1}, G: g_{0} \simeq g_{1}$ and there exists a $V$ such that $V \cap \operatorname{Coin}(F, G)$ is compact, then $\mathbf{S}_{0}=V \cap \operatorname{Coin}\left(f_{0}, g_{0}\right)$ and $V \cap \mathbf{S}_{1}=\operatorname{Coin}\left(f_{1}, g_{1}\right)$ have $\operatorname{Ind}\left(f_{0}, g_{0}, \mathbf{S}_{0}\right)=\operatorname{Ind}\left(f_{1}, g_{1}, \mathbf{S}_{1}\right)$.

3. Additivity: if $\mathbf{S}=\bigcup \mathbf{S}_{i}$, then $\operatorname{Ind}(f, g, \mathbf{S})=\sum \operatorname{Ind}\left(f, g, \mathbf{S}_{i}\right)$.

4. Products: Given $f, g: M_{1} \rightarrow M_{2}$ and $f^{\prime}, g^{\prime}: M_{1}^{\prime} \rightarrow M_{2}^{\prime}$ and $\mathbf{S} \subseteq \operatorname{Coin}(f, g), \mathbf{S}^{\prime} \subseteq \operatorname{Coin}\left(f^{\prime}, g^{\prime}\right)$, then $\operatorname{Ind}(f, g, \mathbf{S})$ and $\operatorname{Ind}\left(f^{\prime}, g^{\prime}, \mathbf{S}^{\prime}\right)$ are defined if and only if $\operatorname{Ind}\left(f \times f^{\prime}, g \times g^{\prime}, \mathbf{S} \times \mathbf{S}^{\prime}\right)$ is defined. If all are defined, $\operatorname{Ind}\left(f \times f^{\prime}, g \times g^{\prime}, \mathbf{S} \times \mathbf{S}^{\prime}\right)=\operatorname{Ind}(f, g, \mathbf{S})$. $\operatorname{Ind}\left(f^{\prime}, g^{\prime}, \mathbf{S}^{\prime}\right)$.

A coincidence class $\mathbf{S}$ is algebraically essential if $\operatorname{Ind}(f, g, \mathbf{S}) \neq 0$. Of course, an algebraically essential class is topologically essential: if $f^{\prime} \simeq f$ and $g^{\prime} \simeq g$, then the corresponding class $\mathbf{S}^{\prime} \neq \varnothing$. Conversely, if $\operatorname{dim}\left(M_{i}\right) \geq 3$, then for every $f$ and $g$, there exist $f^{\prime} \simeq f$ and $g^{\prime} \simeq g$ such that, for each coincidence class $\mathbf{S}$ of $f$ and $g$, the corresponding class $\mathbf{S}^{\prime}$ consists of a single point if $\mathbf{S}$ is algebraically essential and is empty if $\mathbf{S}$ is algebraically inessential [22]. Thus the Nielsen number can be defined as the number of algebraically essential coincidence classes. 
Contrasting with the Nielsen number is an algebraic count of the essential classes: $\operatorname{Ind}(f, g)=\sum \operatorname{Ind}(f, g, \mathbf{S})$, the sum of the coincidence index over all coincidence classes. Of course, this can be computed directly, using $W=V=M_{1}$ to generate $(f, g)_{\bullet}$. This is also a homotopy invariant, but it is not in general a good estimate on the number of essential coincidence classes. This for two reasons. First, there can be cancellation. If $\operatorname{Coin}(f, g)$ has two coincidence classes $\mathbf{S}_{1}, \mathbf{S}_{2}$ with $\operatorname{Ind}\left(f, g, \mathbf{S}_{1}\right)=1$ and $\operatorname{Ind}\left(f, g, \mathbf{S}_{2}\right)=-1$, then every $f^{\prime} \simeq f$ and $g^{\prime} \simeq g$ have at least two coincidence points, yet $\operatorname{Ind}(f, g)=0$ detects none. Second, there can be multiplicity. If Coin $(f, g)$ consists of a single point $c$ with $\operatorname{Ind}(f, g, c)=2$, then $\operatorname{Ind}(f, g)=\operatorname{Ind}(f, g, c)$ overestimates the number of coincidences of $f$ and $g$. However, it is at least true that if $\operatorname{Ind}(f, g) \neq 0$, then $N(f, g) \neq 0$ and $\operatorname{Coin}(f, g) \neq \varnothing$.

Moreover, Ind $(f, g)$ is relatively easy to compute. In rational coefficients, let $D_{i}: H^{p}\left(M_{i}\right) \rightarrow H_{n-p}\left(M_{i}\right)$ be the duality isomorphism and let $\Theta_{p}(f, p)$ be the composition

$$
H_{p}\left(M_{1}\right) \stackrel{f_{*}}{\rightarrow} H_{p}\left(M_{2}\right) \stackrel{D_{2}^{-1}}{\rightarrow} H^{n-p}\left(M_{2}\right) \stackrel{g^{*}}{\rightarrow} H^{n-p}\left(M_{1}\right) \stackrel{D_{1}}{\rightarrow} H_{p}\left(M_{1}\right) .
$$

The Lefschetz coincidence number $L(f, g)$ is defined as

$$
\sum_{p=0}^{n}(-1)^{p} \operatorname{tr} \Theta_{p}(f, g),
$$

and the Lefschetz coincidence theorem states that $L(f, g)=$ $\operatorname{Ind}(f, g)$.

3. Coincidence numbers vs. fixed-point numbers. The coincidence theory outlined above is derived from the analogous form of the Nielsen and Lefschetz fixed-point theories. However, it is not strictly speaking a generalization of the fixed-point theory. That is, it is not clear that the fixed-point theory is recovered by setting $X_{1}=X_{2}$ and $g=$ id. For Nielsen numbers, there may be a difference between $N(f)$ and $N(f, \mathrm{id})$ : in the definition of $N(f, \mathrm{id})$, both $f$ and id are allowed to be modified by a homotopy; while in the definition of $N(f)$, only the map $f$ is. For Lefschetz numbers, the coincidence number $L(f, g)$ is defined only for orientable manifolds, while the fixed point number $L(f)$ is defined for all polyhedra. All of these (potential) global differences have corresponding local differences: A set may be inessential as a coincidence class of $(f, \mathrm{id})$ yet essential 
as a fixed-point class of $f$. Similarly, $L(f, g)$ is only defined for orientable manifolds because the coincidence index is only defined in that setting. The goal of this section is to remove some of these apparent differences between fixed-point theory and coincidence theory.

LEMMA 3.1. If $X$ is a compact orientable manifold, $\mathbf{S}$ an isolated fixed-point set for $f: X \rightarrow X$, then $\operatorname{Ind}(f, \mathrm{id}, \mathbf{S})=\operatorname{index}(f, \mathbf{S})$, where index $(f, \mathbf{S})$ is the fixed-point index of $\mathbf{S}$.

COROLlary 3.2. If $X$ is a compact orientable manifold, then $L(f, \mathrm{id})=L(f)$ for all $f: X \rightarrow X$.

THEOREM 3.3 [5, Cor. 3]. If $X$ is a compact manifold, then $N(f$,id $)$ $=N(f)$ for all $f: X \rightarrow X$.

Though not employing the language of Nielsen coincidence numbers, [10] and [13] contain similar results.

The following lemma helps to generalize these results, and the definition of the coincidence index, from $g=$ id to any homeomorphism $g$.

LemMA 3.4. Suppose $X_{0}, X_{1}, X_{2}, X_{3}$ are compact polyhedra, $h_{0}$ : $X_{0} \rightarrow X_{1}$ and $h_{1}: X_{2} \rightarrow X_{3}$ are homeomorphisms. Then for all $f, g:$ $X_{1} \rightarrow X_{2}$, there is a bijection $\bar{h}_{0}: \mathscr{R}\left(h_{1} \circ f \circ h_{0}, h_{1} \circ g \circ h_{0}\right) \rightarrow \mathscr{R}(f, g)$ with $\bar{h}_{0}\left(\mathscr{E}\left(h_{1} \circ f \circ h_{0}, h_{1} \circ g \circ h_{0}\right)\right)=\mathscr{E}(f, g)$, and which preserves indices up to a sign (when defined).

Proof. The homeomorphism $h_{0}$ restricts to a homeomorphism

$$
h_{0}: \operatorname{Coin}\left(h_{1} \circ f \circ h_{0}, h_{1} \circ g \circ h_{0}\right) \rightarrow \operatorname{Coin}(f, g) \text {. }
$$

If $\mathbf{S}_{0} \in \mathscr{R}\left(h_{1} \circ f \circ h_{0}, h_{1} \circ g \circ h_{0}\right)$ and $x_{0}, x_{1} \in \mathbf{S}_{0}$, then there is a path $\omega$ in $X_{0}$ from $x_{0}$ to $x_{1}$ with $h_{1} \circ f \circ h_{0} \circ \omega \simeq h_{1} \circ g \circ h_{0} \circ \omega$. Thus $h_{0}\left(\mathbf{S}_{0}\right)$ is contained in a single coincidence class $\mathbf{S}_{1}$ of $f$ and $g$. Similarly, $\mathbf{S}_{1}$ maps under $h_{0}^{-1}$ into a single coincidence class of $h_{1} \circ f \circ h_{0}$ and $h_{1} \circ g \circ h_{0}$, that is, into $\mathbf{S}_{0}$.

If $\mathbf{S}_{1}$ is removed by homotopies $F: f \simeq f^{\prime}$ and $G: g \simeq g^{\prime}$, then $\mathbf{S}_{0}$ is removed by homotopies $h_{1} \circ F \circ h_{0}: h_{1} \circ f \circ h_{0} \simeq h_{1} \circ f^{\prime} \circ h_{0}$ and $h_{1} \circ G \circ h_{0}: h_{1} \circ g \circ h_{0} \simeq h_{1} \circ g^{\prime} \circ h_{0}$, and conversely.

Finally, suppose indices are defined for both $(f, g)$ and $\left(h_{1} \circ f \circ\right.$ $\left.h_{0}, h_{1} \circ g \circ h_{0}\right)$. Then either $g=\mathrm{id}$ and $h_{1}=h_{0}^{-1}$, or all of the spaces involved are orientable manifolds. In the first case, both of the indices 
involved are fixed-point indices, and the result is a standard one. If all of the spaces are orientable, then $\left(h_{1} \circ f \circ h_{0}, h_{1} \circ g \circ h_{0}\right)$. is given by the composition

$$
\begin{aligned}
H_{n}\left(X_{0}\right) \rightarrow H_{n}\left(X_{1}\right) \stackrel{(f, g)}{\stackrel{(f,}{\longrightarrow}} H_{n}\left(X_{2} \times X_{2}, X_{2} \times X_{2} \backslash \Delta\left(X_{2}\right)\right) \\
\stackrel{\left(h_{1} \times h_{1}\right)}{\longrightarrow} \cdot H_{n}\left(X_{3} \times X_{3}, X_{3} \times X_{3} \backslash \Delta\left(X_{3}\right)\right)
\end{aligned}
$$

and so differs from $(f, g)$. by (at most) $\operatorname{deg}\left(h_{0}\right) \cdot \operatorname{deg}\left(h_{1}\right)$.

This motivates an extension of the coincidence index: Suppose $X_{1}, X_{2}$ are compact polyhedra, and $f, g: X_{1} \rightarrow X_{2}$ are maps with $g$ a homeomorphism. If $\mathbf{S}$ is an isolated coincidence set, define $\operatorname{Ind}(f, g, \mathbf{S}) \equiv \operatorname{index}\left(g^{-1} \circ f, \mathbf{S}\right)$. Clearly, this inherits all of the standard properties of the index, and agrees with the usual definition of $\operatorname{Ind}(f, g, \mathbf{S})$ when $X_{1}$ and $X_{2}$ are orientable manifolds. To save tedious repetition, we will assume from now on that whenever the coincidence index is involved, either one of the maps is a homeomorphism, or both of the spaces are compact orientable manifolds of the same dimension. We will consider the utility of this definition in $\S \S 5$ and 7.

Corollary 3.5. Suppose $X_{0}, X_{1}, X_{2}, X_{3}$ are compact polyhedra, $h_{0}: X_{0} \rightarrow X_{1}$ and $h_{1}: X_{2} \rightarrow X_{3}$ are homeomorphisms. Then

$$
N\left(h_{1} \circ f \circ h_{0}, h_{1} \circ g \circ h_{0}\right)=N(f, g) \text { for all } f, g: X_{1} \rightarrow X_{2} \text {. }
$$

In particular, if $g: X_{1} \rightarrow X_{2}$ is a homeomorphism between compact polyhedra, then

$$
N\left(f \circ g^{-1}, \mathrm{id}\right)=N(f, g)=N\left(g^{-1} \circ f, \text { id }\right) \text { for all } f: X_{1} \rightarrow X_{2} \text {. }
$$

Corollary 3.6. If $g: M \rightarrow M$ is a homeomorphism between compact manifolds, then $N\left(f \circ g^{-1}\right)=N(f, g)=N\left(g^{-1} \circ f\right)$ for all $f: M \rightarrow M$.

Corollary 3.7. Suppose $X_{1}$ and $X_{2}$ are compact orientable $n$ manifolds and $g$ is a homotopy equivalence with homotopy inverse $g^{\prime}$. Choose orientations for $X_{1}$ and $X_{2}$ so that $\operatorname{deg}(g)=\operatorname{deg}\left(g^{\prime}\right)=1$. Then $L(f, g)=L\left(g^{\prime} \circ f\right)=L\left(f \circ g^{\prime}\right)$.

4. Covering spaces and lifts. Given $f, g: X_{1} \rightarrow X_{2}$, we want to relate the Nielsen and Lefschetz numbers of $f$ and $g$ to Nielsen and Lefschetz numbers of lifts $\tilde{f}, \tilde{g}: \widetilde{X}_{1} \rightarrow \widetilde{X}_{2}$, where $\widetilde{X}_{1}$ and $\widetilde{X}_{2}$ are finite covers of $X_{1}$ and $X_{2}$. 
Fix base points $x_{1} \in X_{1}, x_{2} \in X_{2}$, and for convenience assume that $f\left(x_{1}\right)=x_{2}=g\left(x_{1}\right)$. Let $\pi_{i}$ denote $\pi\left(X_{i}, x_{i}\right)$ and define $\mathscr{C}\left(\pi_{i}\right)=\left\{\Gamma \triangleleft \pi_{i} \mid\left[\pi_{i}: \Gamma\right]<\infty\right\}$. There is a one-to-one correspondence between elements of $\mathscr{C}\left(\pi_{i}\right)$ and finite regular covers of $X_{i}$. Because the Lefschetz coincidence number requires orientable manifolds, we will be particularly interested in finding orientable covering spaces when $X_{1}$ and $X_{2}$ are manifolds. Recall that any manifold has an orientable cover. This cover corresponds to the subgroup $O_{i}=O\left(\pi_{i}\right) \triangleleft \pi_{i}$ consisting of $\alpha \in \pi_{i}$ such that the tangent space map $D \tau_{\alpha}: T_{x_{i}} X_{i} \rightarrow T_{x_{i}} X_{i}$ induced by translation along $\alpha$ acts orientably (i.e. has positive determinant). $O_{i}=\pi_{i}$ if $X_{i}$ is orientable, and is a subgroup of index 2 if $X_{i}$ is nonorientable. Then the orientable finite regular covers correspond to $\mathscr{C} \mathscr{O}\left(\pi_{i}\right)=\left\{\Gamma \in \mathscr{C}\left(\pi_{i}\right) \mid \Gamma \subseteq O_{i}\right\}$.

Now consider $f, g: X_{1} \rightarrow X_{2}$. Fix $\Gamma_{2} \in \mathscr{C}\left(\pi_{2}\right)$ and corresponding finite regular cover $p_{2}: \tilde{X}_{2} \rightarrow X_{2}$. Given a cover $p_{1}: \widetilde{X}_{1} \rightarrow X_{1}$ and corresponding $\Gamma_{1} \in \mathscr{C}\left(\pi_{1}\right), f$ and $g$ lift to some $\tilde{f}, \tilde{g}: \widetilde{X}_{1} \rightarrow \widetilde{X}_{2}$ if and only if $f_{\#}, g_{\#}: \pi_{1} \rightarrow \pi_{2}$ have $f_{\#}, g_{\#}\left(\Gamma_{1}\right) \subseteq \Gamma_{2}$. So define $\mathscr{C}\left(f, g, \Gamma_{2}\right)=\left\{\Gamma_{1} \in \mathscr{C}\left(\pi_{1}\right) \mid f_{\#}, g_{\#}\left(\Gamma_{1}\right) \subseteq \Gamma_{2}\right\}$. Note that $f_{\#}^{-1}\left(\Gamma_{2}\right) \cap$ $g_{\#}^{-1}\left(\Gamma_{2}\right) \in \mathscr{C}\left(f, g, \Gamma_{2}\right)$, so $\mathscr{C}\left(f, g, \Gamma_{2}\right)$ is nonempty. We will refer to the lifting diagram

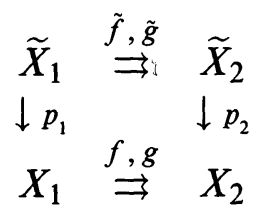

as the $\Gamma_{1}-\Gamma_{2}$ lifting diagram of $f$ and $g$.

For any lifting diagram, the lifts $\tilde{f}, \tilde{g}$ have a Nielsen coincidence number defined. However, they may not have a coincidence index defined, even if $f$ and $g$ did. That is, if the spaces are not manifolds but $g$ is a homeomorphism, they have a coincidence index defined. Then $\tilde{f}$ and $\tilde{g}$ will only have an index defined if $\Gamma_{1}=g_{\#}^{-1}\left(\Gamma_{2}\right)$ has $f_{\#}\left(\Gamma_{1}\right) \subseteq \Gamma_{2}$. But of course, this may not be the case for all $f$ and $g$. We therefore define for every $\Gamma_{2} \in \mathscr{C}\left(\pi_{2}\right)$ the set

$$
\begin{aligned}
\mathscr{C}\left(f, g, \Gamma_{2}\right)=\left\{\Gamma_{1} \in \mathscr{C}\left(\pi_{1}\right) \mid f_{\#}\left(\Gamma_{1}\right), g_{\#}\left(\Gamma_{1}\right) \subseteq \Gamma_{2}\right. \\
\left.\quad \text { and an index is defined for the } \Gamma_{1}-\Gamma_{2} \text { lifts }\right\} .
\end{aligned}
$$

If $X_{1}$ and $X_{2}$ are manifolds and $\Gamma_{2} \in \mathscr{C O}\left(\pi_{2}\right)$, then $f_{\#}^{-1}\left(\Gamma_{2}\right) \cap$ $g_{\#}^{-1}\left(\Gamma_{2}\right) \cap O_{1} \in \mathscr{C}\left(f, g, \Gamma_{2}\right)$, so $\mathscr{C}\left(f, g, \Gamma_{2}\right)$ is nonempty. If $X_{1}$ and $X_{2}$ are not manifolds, or if $\Gamma_{2} \notin \mathscr{C} \mathscr{O}\left(\pi_{2}\right)$, then $\mathscr{C}\left(f, g, \Gamma_{2}\right)$ is nonempty if and only if there is a $\Gamma_{1} \in \mathscr{C}\left(\pi_{1}\right)$ with $f_{\#}\left(\Gamma_{1}\right) \subseteq \Gamma_{2}$ and $g_{\#}$ an isomorphism from $\Gamma_{1}$ to $\Gamma_{2}$. 
In any $\Gamma_{1}-\Gamma_{2}$ lifting diagram, $\Gamma_{i}$ has covering group $\Phi_{i}=\pi_{i} / \Gamma_{i}$. $f_{\#}$ and $g_{\#}$ induce maps $\bar{f}, \bar{g}: \Phi_{1} \rightarrow \Phi_{2}$. If $\tilde{f}, \tilde{g}: \widetilde{X}_{1} \rightarrow \widetilde{X}_{2}$ are fixed "reference lifts" of $f$ and $g$, then all lifts have the form $\beta \circ \tilde{f} \circ \alpha, \beta \circ$ $\tilde{g} \circ \alpha$ with $\alpha \in \Phi_{1}, \beta \in \Phi_{2}$. But $\tilde{f} \circ \alpha=\bar{f}(\alpha) \circ \tilde{f}$, so we need only consider lifts of the form $\beta \circ \tilde{f}, \beta \circ \tilde{g}$.

This has all been done relative to fixed base points $x_{1} \in X_{1}, x_{2} \in$ $X_{2}$. However, the sets are really base point-independent. Consider $x_{1}, x_{1}^{\prime} \in \operatorname{Coin}(f, g)$, with $x_{2}=f\left(x_{1}\right), x_{2}^{\prime}=f\left(x_{1}^{\prime}\right)$. Fix a path $\gamma$ from $x_{1}$ to $x_{1}^{\prime}$. Then there are commutative diagrams

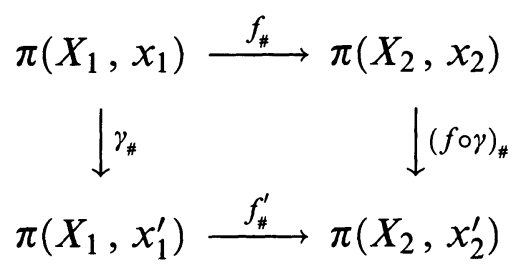

and

$$
\begin{array}{cr}
\pi\left(X_{1}, x_{1}\right) \stackrel{g_{\#}}{\longrightarrow} & \pi\left(X_{2}, x_{2}\right) \\
\downarrow \gamma_{\#} & \\
\pi\left(X_{1}, x_{1}^{\prime}\right) \stackrel{g_{\#}^{\prime}}{\longrightarrow} \pi\left(X_{2}, x_{2}^{\prime}\right) .
\end{array}
$$

The maps $(f \circ \gamma)_{\#}$ and $(g \circ \gamma)_{\#}$ differ by an inner automorphism, so if $\Gamma_{2} \in \mathscr{C}\left(\pi\left(X_{2}, x_{2}\right)\right),(f \circ \gamma)_{\#}\left(\Gamma_{2}\right)$ and $(g \circ \gamma)_{\#}\left(\Gamma_{2}\right)$ are both normal in $\pi\left(X_{2}, x_{2}^{\prime}\right)$, and so are equal. That is, there is a well defined bijection between $\mathscr{C}\left(\pi\left(X_{2}, x_{2}\right)\right)$ and $\mathscr{C}\left(\pi\left(X_{2}, x_{2}^{\prime}\right)\right)$, and a similar bijection between $\mathscr{C}\left(\pi\left(X_{1}, x_{1}\right)\right)$ and $\mathscr{C}\left(\pi\left(X_{1}, x_{1}^{\prime}\right)\right)$. From the diagrams above, these bijections preserve the set $\mathscr{C}\left(f, g, \Gamma_{2}\right)$.

If $X_{i}$ is a manifold, the bijection between $\mathscr{C}\left(\pi\left(X_{i}, x_{i}\right)\right)$ and $\mathscr{C}\left(\pi\left(X_{i}, x_{i}^{\prime}\right)\right)$ preserves the orientation subgroup $O_{i}$ and the set $\mathscr{C O O}\left(\pi_{i}\right)$. So if the $\Gamma_{1}-\Gamma_{2}$ lifting diagram has an index defined because $\Gamma_{i} \in \mathscr{C O}\left(\pi_{i}\right)$, this is independent of the base-point. On the other hand, if the index is defined because $g_{\#}$ is an isomorphism from $\Gamma_{1}$ to $\Gamma_{2}$, then this too is a base-point independent property. That is, the bijection between $\mathscr{C}\left(\pi\left(X_{1}, x_{1}\right)\right)$ and $\mathscr{C}\left(\pi\left(X_{1}, x_{1}^{\prime}\right)\right)$ preserves the set $\mathscr{I C}\left(f, g, \Gamma_{2}\right)$.

The ability to construct lifts, or lifts which admit an index, is then independent of the base-point chosen. We now turn to the coincidence theory for lifts. We will see that here, the choice of base-point is significant. 
5. Coincidence numbers for covering spaces. We now consider the relation between the coincidence sets, Nielsen numbers and Lefschetz numbers of $f$ and $g$ and those of the lifts. Much of this is simply a modification of the universal cover/lifting approach to Nielsen numbers, as found in [15].

Proposition 5.1. S is a coincidence class in $\operatorname{Coin}(\tilde{f}, \tilde{g})$ if and only if it is a coincidence class in $\operatorname{Coin}(\beta \circ \tilde{f}, \beta \circ \tilde{g})$ for all $\beta \in \Phi_{2}$.

Proposition 5.2. If $\tilde{x} \in \operatorname{Coin}(\beta \circ \tilde{f}, \tilde{g})$ for some $\beta \in \Phi_{2}$, then $x=p_{1}(\tilde{x}) \in \operatorname{Coin}(f, g)$. Conversely, if $x \in \operatorname{Coin}(f, g)$ and $\tilde{x} \epsilon$ $p^{-1}(x)$, then there is a unique $\beta \in \Phi_{2}$ such that $\tilde{x} \in \operatorname{Coin}(\beta \circ \tilde{f}, \tilde{g})$. That is,

$$
p_{1}^{-1}(\operatorname{Coin}(f, g))=\bigsqcup_{\beta \in \Phi_{2}} \operatorname{Coin}(\beta \circ \tilde{f}, \tilde{g}) \text {. }
$$

Proof. If $\tilde{x} \in \operatorname{Coin}(\beta \circ \tilde{f}, \tilde{g})$, then $f(x)=f \circ p_{1}(\tilde{x})=p_{2} \circ \tilde{f}(\tilde{x})=$ $p_{2} \circ \tilde{g}(\tilde{x})=g \circ p_{1}(\tilde{x})=g(x)$. If $p_{2} \circ \tilde{f}(\tilde{x})=f \circ p_{1}(\tilde{x})=f(x)=g(x)=$ $g \circ p_{1}(\tilde{x})=p_{2} \circ \tilde{g}(\tilde{x})$, then $\tilde{g}(\tilde{x})$ and $\tilde{f}(\tilde{x})$ lie in the same fiber, and there is a unique $\beta \in \Phi_{2}$ so that $\tilde{g}(\tilde{x})=\beta \circ \tilde{f}(\tilde{x})$.

Proposition 5.3. If $\widetilde{\mathbf{S}}$ is a coincidence class in $\operatorname{Coin}(\tilde{f}, \tilde{g})$ and $\alpha \in \Phi_{1}$, then $\mathbf{S}=p_{1}(\widetilde{\mathbf{S}})$ is a coincidence class in $\operatorname{Coin}(f, g)$ and $\alpha(\widetilde{\mathbf{S}})$ is a coincidence class in $\operatorname{Coin}(\beta \circ \tilde{f}, \tilde{g})$, where $\beta=\bar{g}(\alpha) \cdot \bar{f}^{-1}(\alpha)$.

Proof. If $\tilde{x}_{1}, \tilde{x}_{2} \in \widetilde{\mathbf{S}}$, then there is a path $\tilde{\boldsymbol{\omega}}$ in $\widetilde{X}_{1}$ from $\tilde{x}_{1}$ to $\tilde{x}_{2}$ with $\tilde{f} \circ \tilde{\omega} \simeq \tilde{g} \circ \tilde{\omega}$. If $x_{i}=p_{1}\left(\tilde{x}_{i}\right)$, then $\omega=p_{1} \tilde{\omega}$ is a path from $x_{1}$ to $x_{2}$ with $H: f \circ \omega \simeq g \circ \omega$. Conversely, suppose $x_{1}, x_{2} \in \operatorname{Coin}(f, g)$ are connected by a path $\omega$ with $f \circ \omega \simeq g \circ \omega$. If $\tilde{x}_{1} \in \operatorname{Coin}(\tilde{f}, \tilde{g})$ lies over $x_{1}$, let $\tilde{\omega}$ be the unique lift of $\omega$ based at $\tilde{x}_{1}$, and let $\widetilde{H}$ be the unique lift of $H$ based at $\tilde{f}\left(\tilde{x}_{1}\right)$. Let $\tilde{x}_{2}=\tilde{\omega}(1)$. Then $\tilde{f}\left(\tilde{x}_{2}\right)=\tilde{g}_{2}\left(\tilde{x}_{2}\right)$ and $\tilde{H}: \tilde{f} \circ \tilde{\omega} \simeq \tilde{g} \circ \tilde{\omega}$.

Now, if $\tilde{x} \in \widetilde{\mathbf{S}}$ and $\alpha \in \Phi_{1}$, then $\tilde{f}(\alpha(\tilde{x}))=\bar{f}(\alpha) \circ \tilde{f}(\tilde{x})$ and $\tilde{g}(\alpha(\tilde{x}))=\bar{g}(\alpha) \circ \tilde{g}(\tilde{x})$. Thus $\beta \circ \tilde{f}(\alpha(\tilde{x}))=\tilde{g}(\alpha(\tilde{x}))$ if and only if $\beta=\bar{g}(\alpha) \cdot \bar{f}^{-1}(\alpha)$. And if $\widetilde{H}: \tilde{f} \circ \tilde{\omega} \simeq \tilde{g} \circ \tilde{\omega}$, then $\bar{g}(\alpha) \circ \tilde{H}$ : $\beta \circ \tilde{f} \circ \alpha_{-1} \tilde{\omega} \simeq \tilde{g} \circ \alpha_{-1} \tilde{\omega}$, so $\alpha(\widetilde{\mathbf{S}})$ is a coincidence class in $\operatorname{Coin}(\beta \circ \tilde{f}, \tilde{g})$.

If $\mathbf{S}$ is a coincidence class of $f$ and $g$, then define $C_{\#}(f, g, \mathbf{S})=$ $\left\{\alpha \in \pi_{1} \mid f_{\#}(\alpha)=g_{\#}(\alpha)\right\}$, where $f_{\#}$ and $g_{\#}$ are based at some $x \in \mathbf{S}$. $C_{\#}(f, g, \mathbf{S})$ is a subgroup of $\pi_{1}$, but is not necessarily normal. Note 
that $C_{\#}(f, g, \mathbf{S})$ depends on $\mathbf{S}$, but not on the base point $x \in \mathbf{S}$ chosen. Similarly, if $X_{1}$ and $X_{2}$ are manifolds of the same dimension, let $C O_{\#}(f, g, \mathbf{S})=C_{\#}(f, g, \mathbf{S}) \cap O\left(\pi_{1}\right)$.

Corollary 5.4. If $\widetilde{\mathbf{S}}$ is a coincidence class in $\operatorname{Coin}(\beta \circ \tilde{f}, \tilde{g})$, then $\widetilde{\mathbf{S}}$ is covering space over $\mathbf{S}$ with deck transformations

$$
C_{\#}(f, g, \mathbf{S}) / \Gamma_{1} \cap C_{\#}(f, g, \mathbf{S}) .
$$

That is, if $\mathbf{S}$ is a coincidence class in $\operatorname{Coin}(f, g)$, then $\Phi_{1}$ acts transitively on the coincidence classes in $p^{-1}(\mathbf{S})$ with isotropy group of $C_{\#}(f, g, \mathbf{S}) \cdot \Gamma_{1} / \Gamma_{1}$. Thus $\mathbf{S}$ is covered by $\left[\pi_{1}: C_{\#}(f, g, \mathbf{S}) \cdot \Gamma_{1}\right]$ coincidence classes. In particular, the number of classes covering $\mathbf{S}$ depends on $\mathbf{S}$.

Of course, to compute the Nielsen number of lifts $\tilde{f}$ and $\tilde{g}$, we must consider more than just the number of coincidence classes. We must also consider which classes are essential and which are inessential. The ideal situation would be that a class $\mathbf{S} \in \mathscr{R}(f, g)$ is essential if and only if all of the classes $\widetilde{\mathbf{S}}$ covering it are. This is almost, but not quite, true.

Proposition 5.5. If $\widetilde{\mathbf{S}}$ is an essential coincidence class in $\operatorname{Coin}(\tilde{f}, \tilde{g}), \alpha \in \Phi_{1}$, then $\mathbf{S}=p_{1}(\widetilde{\mathbf{S}})$ is an essential coincidence class in $\operatorname{Coin}(f, g)$ and $\alpha(\widetilde{\mathbf{S}})$ is an essential coincidence class in $\operatorname{Coin}(\beta \circ \tilde{f}, \tilde{g})$, where $\beta=\bar{g}(\alpha) \circ(\bar{f}(\alpha))^{-1}$.

Proof. Suppose $\mathbf{S}$ is inessential. Then there exist $f^{\prime} \simeq f$ and $g^{\prime} \simeq g$ such that $\mathbf{S}$ continues to an empty coincidence class $\mathbf{C}^{\prime}$. Now lift $f^{\prime} \simeq f$ and $g^{\prime} \simeq g$ to $\tilde{f}^{\prime} \simeq \tilde{f}$ and $\tilde{g}^{\prime} \simeq g^{\prime}$. The class $\widetilde{\mathbf{S}}$ continues to a class $\widetilde{\mathbf{C}}^{\prime}$ which covers $\mathbf{C}^{\prime}$, and so is empty. Thus $\widetilde{\mathbf{S}}$ is inessential. Similarly, if $\alpha(\widetilde{\mathbf{S}})$ is inessential, then the homotopy which removes it can be carried by the deck transformations to a homotopy which removes $\widetilde{\mathbf{S}}$.

Thus every essential class in $\operatorname{Coin}(f, g)$ is covered by either no essential classes, or $\left[\pi_{1}: \Gamma_{1} \cdot C_{\#}(f, g, \mathbf{S})\right]$ essential classes, as $\beta$ ranges over $\Phi_{2}$. Intuitively, if $\mathbf{S}$ is essential, a class $\widetilde{\mathbf{S}}$ in $\operatorname{Coin}(\tilde{f}, \tilde{g})$ covering $\mathbf{S}$ is inessential if it can be removed via homotopies $\widetilde{F}: \tilde{f} \simeq \tilde{f}^{\prime}$ and $\widetilde{G}: \tilde{g} \simeq \tilde{g}^{\prime}$ which are not equivariant under the covering group actions, and so do not project to homotopies on $X_{1}$. It is difficult to find topological conditions that eliminate this possibility. The 
following result, though restricted to the manifold setting, will prove to be sufficient for our purposes.

THEOREM 5.6. Suppose $X_{1}, X_{2}$ are compact manifolds of the same dimension, and neither is a surface with negative Euler characteristic. Suppose $\mathbf{S}$ is an essential coincidence class in $\mathscr{E}(f, g)$.

1. If $C_{\#}(f, g, \mathbf{S}) \subseteq \Gamma_{1}$, then all coincidence classes covering $\mathbf{S}$ in the $\Gamma_{1}-\Gamma_{2}$ lifting diagram of $f$ and $g$ are essential.

2. If $\Gamma_{1} \in \mathscr{I}\left(f, g, \Gamma_{2}\right)$, then all coincidence classes covering $\mathbf{S}$ in the $\Gamma_{1}-\Gamma_{2}$ lifting diagram of $f$ and $g$ are essential if and only if

$$
\begin{aligned}
& O_{1} \cdot \Gamma_{1} / \Gamma_{1} \cap C_{\#}(f, g, \mathbf{S}) \cdot \Gamma_{1} / \Gamma_{1} \\
& \quad=f_{\#}^{-1}\left(O\left(\pi_{2}\right)\right) \cdot \Gamma_{1} / \Gamma_{1} \cap C_{\#}(f, g, \mathbf{S}) \cdot \Gamma_{1} / \Gamma_{1} .
\end{aligned}
$$

Before beginning the proof, some comments on the condition in (2) are in order. The idea is simply that, in moving from one class covering $\mathbf{S}$ to another, the index can only change by changing sign. Changing sign, in turn, occurs when the two covering transformations (or their corresponding elements in the fundamental group) involved in moving one class to another have different "parity"-that is, one changes the orientation, and the other does not. The condition in (2), while awkward, is precisely the condition needed to rule this out.

Proof of Theorem 5.6. The class of manifolds specified is closed under finite covers, and all pairs of manifolds in the class are Wenken pairs. More precisely, the maps $f$ and $g$ (up to homotopy) may be assumed to have each coincidence class contain a single point, which can be removed by a perturbation supported on an arbitrarily small neighborhood if the class is inessential.

First, suppose $C_{\#}(f, g, \mathbf{S}) \subseteq \Gamma_{1}$. Then every coincidence class $\widetilde{\mathbf{S}}$ covering $\mathbf{S}$ consists of a single point. Then any perturbation supported in a neighborhood of it passes down to a perturbation of $f$ and $g$ in a neighborhood of $\mathbf{S}$. If it is inessential and that perturbation removes $\widetilde{\mathbf{S}}$, then the corresponding perturbation removes $\mathbf{S}$ as a coincidence class.

Now suppose $\Gamma_{1} \in \mathscr{C} C\left(f, g, \Gamma_{2}\right)$. We will consider the case $\Gamma_{i} \in$ $\mathscr{C O O}\left(\pi_{i}\right)$ : the case of $\tilde{g}$ a homeomorphism is similar. Suppose $\Gamma_{2} \subseteq$ $O\left(\pi_{2}\right), \Gamma_{1} \in \mathscr{C}\left(f, g, \Gamma_{2}\right)$ and

$$
\begin{aligned}
& O_{1} \cdot \Gamma_{1} / \Gamma_{1} \cap C_{\#}(f, g, \mathbf{S}) \cdot \Gamma_{1} / \Gamma_{1} \\
& \quad=f_{\#}^{-1}\left(O\left(\pi_{2}\right)\right) \cdot \Gamma_{1} / \Gamma_{1} \cap C_{\#}(f, g, \mathbf{S}) \cdot \Gamma_{1} / \Gamma_{1} .
\end{aligned}
$$


If $\tilde{x} \in \widetilde{\mathbf{S}}$, then $\tilde{x}$ is an isolated coincidence, and $\operatorname{Ind}(\tilde{f}, \tilde{g}, \tilde{x})$ is nonzero. Any other element of $\widetilde{\mathbf{S}}$ has the form $\alpha(\tilde{x})$ with $\alpha \in$ $C_{\#}(f, g, \mathbf{S}) \cdot \Gamma_{1} / \Gamma_{1}$. To relate the indices of $\tilde{x}$ and $\alpha(\tilde{x})$ choose neighborhoods $W \subseteq V$ which isolate $\tilde{x}$ as a coincidence. Then $\alpha(W) \subseteq \alpha(V)$ isolate $\alpha(\tilde{x})$, and the indices are related by the diagrams

$$
\begin{array}{ccccc}
H_{n}\left(\widetilde{X}_{1}\right) & \rightarrow & H_{n}\left(\widetilde{X}_{1}, \widetilde{X}_{1} \backslash W\right) & \cong & H_{n}(V, V \backslash W) \\
\alpha_{*} \downarrow & & \alpha_{*} \downarrow & & \alpha_{*} \downarrow \\
H_{n}\left(\widetilde{X}_{1}\right) & \rightarrow & H_{n}\left(\widetilde{X}_{1}, \widetilde{X}_{1} \backslash \alpha(W)\right) & \cong & H_{n}(\alpha(V), \alpha(V) \backslash \alpha(W))
\end{array}
$$

and

$$
\begin{gathered}
H_{n}(V, V \backslash W) \quad \stackrel{\left(f_{*}, g_{*}\right)}{\longrightarrow} H_{n}\left(\widetilde{X}_{2} \times \widetilde{X}_{2}, \widetilde{X}_{2} \times \widetilde{X}_{2} \backslash \Delta\left(\widetilde{X}_{2}\right)\right) \\
\alpha_{*} \downarrow \\
H_{n}(\alpha(V), \alpha(V) \backslash \alpha(W)) \stackrel{\left(f_{*}, g_{*}\right)}{\longrightarrow} H_{n}\left(\widetilde{X}_{2} \times \widetilde{X}_{2}, \widetilde{X}_{2} \times \widetilde{X}_{2} \backslash \Delta\left(\widetilde{X}_{2}\right)\right)
\end{gathered}
$$

so $\operatorname{Ind}(\tilde{f}, \tilde{g}, \alpha(\tilde{x}))=\operatorname{deg}(\alpha) \operatorname{deg}(\bar{f}(\alpha)) \operatorname{Ind}(\tilde{f}, \tilde{g}, \tilde{x})$.

But the condition

$$
\begin{aligned}
& O_{1} \cdot \Gamma_{1} / \Gamma_{1} \cap C_{\#}(f, g, \mathbf{S}) \cdot \Gamma_{1} / \Gamma_{1} \\
& \quad=f_{\#}^{-1}\left(O\left(\pi_{2}\right)\right) \cdot \Gamma_{1} / \Gamma_{1} \cap C_{\#}(f, g, \mathbf{S}) \cdot \Gamma_{1} / \Gamma_{1}
\end{aligned}
$$

is precisely the condition needed to guarantee that $\operatorname{deg}(\alpha)=\operatorname{deg}(\bar{f}(\alpha))$ for $\alpha \in p_{1 \#}\left(C_{\#}(f, g, \mathbf{S})\right)$, so $\operatorname{Ind}(\tilde{f}, \tilde{g}, \alpha(\tilde{x}))=\operatorname{Ind}(\tilde{f}, \tilde{g}, \tilde{x})$. Then

$$
\operatorname{Ind}(\tilde{f}, \tilde{g}, \widetilde{\mathbf{S}})=\left[C_{\#}(f, g, \mathbf{S}) \cdot \Gamma_{1}: \Gamma_{1}\right] \operatorname{Ind}(\tilde{f}, \tilde{g}, \tilde{x}) \neq 0 .
$$

Thus $\widetilde{\mathbf{S}}$ is essential. On the other hand, if the condition fails, exactly half of the elements in $p_{1 \#}\left(C_{\#}(f, g, \mathbf{S})\right)$ will have $\operatorname{deg}(\alpha)=$ $\operatorname{deg}(\bar{f}(\alpha))$, while the other half will have $\operatorname{deg}(\alpha)=-\operatorname{deg}(\bar{f}(\alpha))$. The corresponding points in $\widetilde{\mathbf{S}}$ will have $\operatorname{Ind}(\tilde{f}, \tilde{g}, \alpha(\tilde{x}))=\operatorname{Ind}(\tilde{f}, \tilde{g}, \tilde{x})$ and $\operatorname{Ind}(\tilde{f}, \tilde{g}, \alpha(\tilde{x}))=-\operatorname{Ind}(\tilde{f}, \tilde{g}, \tilde{x})$ respectively, so $\operatorname{Ind}(\tilde{f}, \tilde{g}, \tilde{\mathbf{S}})$ $=0$ and $\mathbf{S}$ is inessential.

Note that if $C_{\#}(f, g, \mathbf{S}) \subseteq \Gamma_{1}$, all coincidence classes covering $\mathbf{S}$ are homeomorphic to $\mathbf{S}$.

Corollary 5.7. If $\pi_{1} \in \mathscr{G}\left(f, g, \pi_{2}\right)$, then for every $\Gamma_{1}-\Gamma_{2}$ lifting diagram, essential classes lift to essential classes. Moreover, if $\mathbf{S} \in \mathscr{R}(f, g)$ and $\mathscr{S}$ is the set of coincidence classes covering $\mathbf{S}$ in the $\Gamma_{1}-\Gamma_{2}$ lifting diagram, then $\left|\Phi_{1}\right| \operatorname{Ind}(f, g, \mathbf{S})=\sum_{\widetilde{\mathbf{S}} \in \mathscr{S}} \operatorname{Ind}(\beta \circ \tilde{f}, \tilde{g})$.

Proof. If $\pi_{1} \in \mathscr{C} C\left(f, g, \pi_{2}\right)$, then either $\pi_{i}=O\left(\pi_{i}\right)$ for $i=1,2$, or $g$ is a homeomorphism. In the former case, condition (ii) of 
Theorem 5.6 is trivially satisfied for all $f$ and $g$. If $g$ is a homeomorphism, we may assume without loss that $g=\mathrm{id}$. Then $O\left(\pi_{2}\right)=$ $O\left(\pi_{1}\right)$ and $f_{\#}^{-1}\left(O\left(\pi_{2}\right)\right)=g_{\#}^{-1}\left(O\left(\pi_{1}\right)\right)=O\left(\pi_{1}\right)$. In either case, if $x \in \operatorname{Coin}(f, g)$ is an isolated coincidence and $\tilde{x} \in p_{1}^{-1}(x)$ is in $\operatorname{Coin}(\tilde{f}, \tilde{g})$, then $\operatorname{Ind}(\tilde{f}, \tilde{g}, \tilde{x})=\operatorname{Ind}(f, g, x)$. Since $\mathbf{S}$ may be assumed to consist of isolated coincidences,

$$
\begin{aligned}
\left|\Phi_{1}\right| \operatorname{Ind}(f, g, \mathbf{S}) & =\sum_{\tilde{x} \in p_{1}^{-1}(\mathbf{S})} \operatorname{Ind}(\beta \circ \tilde{f}, \tilde{g}, \tilde{x}) \\
& =\sum_{\tilde{\mathbf{S}} \in \mathscr{S}} \operatorname{Ind}(\beta \circ \tilde{f}, \tilde{g}) .
\end{aligned}
$$

It will be useful to note what these results imply in the special case $\Gamma_{2}=\pi_{2}, \Gamma_{1} \neq \pi_{1}$ and $\Gamma_{2} \neq \pi_{2}, \Gamma_{1}=\pi_{1}$. In the first case, $\Gamma_{2}=\pi_{2}$, $\Gamma_{1} \neq \pi_{1}$, the maps $f$ and $g$ each have only one lift: $\tilde{f}=f \circ p_{1}$ and $\tilde{g}=g \circ p_{1}$. If $\mathbf{S} \in \mathscr{R}(f, g)$, then $\mathbf{S}$ is covered by $\left[\pi_{1}: C_{\#}(f, g, \mathbf{S})\right.$. $\left.\Gamma_{1}\right]$ coincidence classes. Each is a covering space over $\mathbf{S}$ of order $\left[C_{\#}(f, g, \mathbf{S}) \cdot \Gamma_{1}: \Gamma_{1}\right]$, and either all are essential or all are inessential. To compare the Lefschetz numbers of $(f, g)$ and $(\tilde{f}, \tilde{g})$, we need $\Gamma_{1}, \pi_{1} \in \mathscr{C} C\left(f, g, \pi_{2}\right)$. This is only possible if both $X_{1}$ and $X_{2}$ are orientable manifolds of the same dimension. Then $\Gamma_{1} \subset \pi_{1}=O\left(\pi_{1}\right)$ and $\pi_{2}=O\left(\pi_{2}\right)$. In particular, the covering map $p_{1}: \widetilde{X}_{1} \rightarrow X_{1}$ has $\operatorname{deg}\left(p_{1}\right)=\left|\Phi_{1}\right|$ and $L(\tilde{f}, \tilde{g})=\operatorname{deg}\left(p_{1}\right) \cdot L(f, g)=\left|\Phi_{1}\right| \cdot L(f, g)$.

On the other hand, if $\Gamma_{2} \neq \pi_{2}, \Gamma_{1}=\pi_{1}$, then there are $\left|\Phi_{2}\right|$ lifts $\beta \circ \tilde{f}$ and $\beta \circ \tilde{g}$ for $f$ and $g$, with $f=p_{2} \circ \beta \tilde{f}$ and $g=p_{2} \circ \beta \tilde{g}$. In this case, the decomposition $p_{1}^{-1}(\operatorname{Coin}(f, g))=\bigcup_{\rho \in \Phi_{2}} \operatorname{Coin}(\beta \circ \tilde{f}, \tilde{g})$, becomes a partition of $\operatorname{Coin}(f, g)$, which preserves coincidence classes. That is, there is a bijection $\mathscr{R}(f, g) \leftrightarrow \bigcup \mathscr{R}(\beta \circ \tilde{f}, \tilde{g})$. In the Wenken manifold setting of Theorem 5.6, this bijection preserves essential classes, so $N(f, g)=\sum_{\beta \in \Phi_{2}} N(\beta \circ \tilde{f}, \tilde{g})$. To compare the Lefschetz numbers of $(f, g)$ and $(\beta \circ \tilde{f}, \tilde{g})$, we now need $\pi_{1} \in \mathscr{C} C\left(f, g, \pi_{2}\right)$ and $\pi_{1} \in \mathscr{C} C\left(f, g, \Gamma_{2}\right)$. Once again, this is only possible if $X_{1}$ and $X_{2}$ are orientable manifolds of the same dimension. In that case, any $\mathbf{S} \in \mathscr{R}(f, g)$ has $\operatorname{Ind}(f, g, \mathbf{S})=\operatorname{deg}\left(p_{2}\right) \cdot \operatorname{Ind}(\beta \circ \tilde{f}, \tilde{g})$, so $L(f, g)=\sum_{\beta \in \Phi_{2}} L(\beta \circ \tilde{f}, \tilde{g})$.

To collate all of this information, we now introduce a Nielsentype coincidence number for the $\Gamma_{1}-\Gamma_{2}$ lifting diagram. The following data is required: maps $f, g: X_{1} \rightarrow X_{2}, \Gamma_{2} \in \mathscr{C}\left(\pi_{2}\right), \Gamma_{1} \in$ 
$\mathscr{C}\left(f, g, \Gamma_{2}\right)$ and lifts $\tilde{f}, \tilde{g}$ of $f$ and $g$. We then define

$$
\tilde{N}\left(f, g, \Gamma_{1}\right)=\frac{1}{\left|\Phi_{1}\right|} \sum_{\beta \in \Phi_{2}} N(\beta \circ \tilde{f}, \tilde{g}) .
$$

If $\Gamma_{1} \in \mathscr{C}\left(f, g, \Gamma_{2}\right)$, we can also define a Lefschetz-type coincidence number

$$
\tilde{L}\left(f, g, \Gamma_{2}\right)=\frac{1}{\left|\Phi_{1}\right|} \sum_{\beta \in \Phi_{2}}|L(\beta \circ \tilde{f}, \tilde{g})| .
$$

Apparently, both numbers depend on both $\Gamma_{1}$ and $\Gamma_{2}$. The following result justifies the notation.

THEOREM 5.8. 1. $\tilde{N}\left(f, g, \Gamma_{1}\right)$ is independent of $\Gamma_{2}$. That is, if $\Gamma_{1} \in \mathscr{C}\left(f, g, \Gamma_{2}\right) \cap \mathscr{C}\left(f, g, \Gamma_{2}^{\prime}\right)$, and $(f, g)$ lift to $(\tilde{f}, \tilde{g})$ and $\left(\tilde{f}^{\prime}, \tilde{g}^{\prime}\right)$ in the $\Gamma_{1}-\Gamma_{2}$ and $\Gamma_{1}-\Gamma_{2}^{\prime}$ lifting diagrams respectively, then

$$
\frac{1}{\left|\Phi_{1}\right|} \sum_{\beta \in \Phi_{2}} N(\beta \circ \tilde{f}, \tilde{g})=\frac{1}{\left|\Phi_{1}\right|} \sum_{\beta^{\prime} \in \Phi_{2}^{\prime}} N\left(\beta^{\prime} \circ \tilde{f}^{\prime}, \tilde{g}^{\prime}\right) \text {. }
$$

2. When defined, $\widetilde{L}\left(f, g, \Gamma_{2}\right)$ is independent of $\Gamma_{1}$. That is, if $\Gamma_{1}, \Gamma_{1}^{\prime} \in \mathscr{G}\left(f, g, \Gamma_{2}\right)$, and $(f, g)$ lift to $(\tilde{f}, \tilde{g})$ and $\left(\tilde{f}^{\prime}, \tilde{g}^{\prime}\right)$ in the $\Gamma_{1}-\Gamma_{2}$ and $\Gamma_{1}^{\prime}-\Gamma_{2}^{\prime}$ lifting diagrams respectively, then

$$
\frac{1}{\left|\Phi_{1}\right|} \sum_{\beta \in \Phi_{2}}|L(\beta \circ \tilde{f}, \tilde{g})|=\frac{1}{\left|\Phi_{1}^{\prime}\right|} \sum_{\beta \in \Phi_{2}}\left|L\left(\beta \circ \tilde{f}^{\prime}, \tilde{g}^{\prime}\right)\right| \text {. }
$$

Proof. To show that $\tilde{N}\left(f, g, \Gamma_{1}\right)$ is independent of $\Gamma_{2}$, suppose $\Gamma_{2}^{\prime} \subset \Gamma_{2}$ and $\Gamma_{1} \in \mathscr{C}\left(f, g, \Gamma_{2}^{\prime}\right)$. We may assume that $\tilde{f}$ and $\tilde{g}$ are covered by $\tilde{f}^{\prime}$ and $\tilde{g}^{\prime}$. If $s: \Phi_{2} \rightarrow \Phi_{2}^{\prime}$ is a section of the natural projection $\Phi_{2}^{\prime} \rightarrow \Phi_{2}$, then for every $\beta \in \Phi_{2}$, the map $s(\beta) \circ \tilde{f}^{\prime}$ can be used as a reference lift for $\beta \circ \tilde{f}$. Let $\Psi_{2}=\Phi_{2}^{\prime} / \Phi_{2}$. Then $N(\beta \circ \tilde{f}, \tilde{g})=\sum_{\psi \in \Psi_{2}} N\left(\psi \circ s(\beta) \circ \tilde{f}^{\prime}, \tilde{g}^{\prime}\right)$, and

$$
\begin{aligned}
\frac{1}{\left|\Phi_{1}\right|} \sum_{\beta \in \Phi_{2}} N(\beta \circ \tilde{f}, \tilde{g}) & =\frac{1}{\left|\Phi_{1}\right|} \sum_{\beta \in \Phi_{2}} \sum_{\psi \in \Psi_{2}} N\left(\psi \circ s(\beta) \circ \tilde{f}^{\prime}, \tilde{g}^{\prime}\right) \\
& =\frac{1}{\left|\Phi_{1}\right|} \sum_{\beta^{\prime} \in \Phi_{2}^{\prime}} N\left(\beta^{\prime} \circ \tilde{f}^{\prime}, \tilde{g}^{\prime}\right) .
\end{aligned}
$$

Of course, if $\Gamma_{2}^{\prime} \not \subset \Gamma_{2}$, both contain $\Gamma_{2}^{\prime} \cap \Gamma_{2}$ and $\Gamma_{1} \in$ $\mathscr{C}\left(f, g, \Gamma_{2}^{\prime} \cap \Gamma_{2}\right)$, so $\Gamma_{2}^{\prime}$ can be replaced by $\Gamma_{2}^{\prime} \cap \Gamma_{2}$. 
To show that $L\left(f, g, \Gamma_{2}\right)$ is independent of $\Gamma_{1}$, suppose $\Gamma_{1}^{\prime} \subset \Gamma_{1}$ and $\Gamma_{1}^{\prime}, \Gamma_{1} \in \mathscr{I C}\left(f, g, \Gamma_{2}\right)$. This requires $\Gamma_{i} \subseteq O\left(\pi_{i}\right)$. Suppose $p_{1}: \tilde{X}_{1} \rightarrow X_{1}$ and $p_{1}^{\prime}: \widetilde{X}_{1}^{\prime} \rightarrow X_{1}$ are the covering spaces corresponding to $\Gamma_{1}$ and $\Gamma_{1}^{\prime}$ respectively, $p: \tilde{X}_{1}^{\prime} \rightarrow \widetilde{X}_{1}$ is the covering map of $\tilde{X}_{1}^{\prime}$ over $\tilde{X}_{1}$, and $\tilde{f}, \tilde{g}$ are lifts of $f$ and $g$ to $\tilde{X}_{1}$. Then $\tilde{f} \circ p$ and $\tilde{g} \circ p$ are the lifts of $f$ and $g$ to $\widetilde{X}_{1}^{\prime}$, and $L(\tilde{f} \circ p, \tilde{g} \circ p)=\operatorname{deg}(p) \cdot L(\tilde{f}, \tilde{g})=$ $\left|\Gamma_{1}: \Gamma_{1}^{\prime}\right| \cdot L(\tilde{f}, \tilde{g})$. Then

$$
\begin{aligned}
\frac{1}{\left|\Phi_{1}\right|} \sum_{\beta \in \Phi_{2}}|L(\beta \circ \tilde{f}, \tilde{g})| & =\frac{1}{\left|\Phi_{1}^{\prime}\right|} \frac{1}{\left|\Gamma_{1}: \Gamma_{1}^{\prime}\right|} \sum_{\beta \in \Phi_{2}}|L(\beta \circ \tilde{f}, \tilde{g})| \\
& =\frac{1}{\left|\Phi_{1}^{\prime}\right|} \sum_{\beta \in \Phi_{2}}\left|L\left(\beta \circ \tilde{f}^{\prime}, \tilde{g}^{\prime}\right)\right| .
\end{aligned}
$$

Note that, in contrast to $\widetilde{L}\left(f, g, \Gamma_{2}\right)$, the quantity

$$
\frac{1}{\left|\Phi_{1}\right|} \sum_{\beta \in \Phi_{2}} L(\beta \circ \tilde{f}, \tilde{g})
$$

(when defined) is independent of both $\Gamma_{1}$ and $\Gamma_{2}$. This is one reason for the introduction of the absolute value in the definition of $\widetilde{L}\left(f, g, \Gamma_{2}\right)$ : without it, no new information is obtained. Also, we note that, in general, $\widetilde{N}$ does depend on $\Gamma_{1}$ and $\widetilde{L}$ does depend on $\Gamma_{2}$. We now examine that dependence.

THEOREM 5.9. 1. If $\Gamma_{1}, \Gamma_{1}^{\prime} \in \mathscr{C}\left(f, g, \Gamma_{2}\right)$ for some $\Gamma_{2} \in \mathscr{C}\left(\pi_{2}\right)$, and $\Gamma_{1}^{\prime} \subseteq \Gamma_{1}$, then $\tilde{N}\left(f, g, \Gamma_{1}\right) \geq \tilde{N}\left(f, g, \Gamma_{1}^{\prime}\right)$.

2. Suppose $X_{1}, X_{2}$ are manifolds of the same dimension, and neither is a surface with negative Euler characteristic. If $C_{\#}(f, g, \mathbf{S}) \cap$ $\Gamma_{1}=C_{\#}(f, g, \mathbf{S}) \cap \Gamma_{1}^{\prime}$ for every $\mathbf{S} \in \mathscr{E}(f, g)$ then $\tilde{N}\left(f, g, \Gamma_{1}\right)=$ $\tilde{N}\left(f, g, \Gamma_{1}^{\prime}\right)$.

Proof. Recall that if $(\tilde{f}, \tilde{g})$ covers $(f, g)$ in the $\Gamma_{1}-\Gamma_{2}$ lifting diagram, and $p: \widetilde{X}_{1}^{\prime} \rightarrow \widetilde{X}_{1}^{\prime}$ is the covering space corresponding to $\Gamma_{1}^{\prime} \rightarrow \Gamma_{1}$, then $(\tilde{f} \circ p, \tilde{g} \circ p)$ covers $(f, g)$ in the $\Gamma_{1}^{\prime}-\Gamma_{2}$ lifting diagram. Then $\widetilde{\mathbf{S}} \in \mathscr{E}(\tilde{f}, \tilde{g})$ is covered by $\left[\Gamma_{1}: C_{\#}(\tilde{f}, \tilde{g}, \widetilde{\mathbf{S}}) \cdot \Gamma_{1}^{\prime}\right]=$ $\left[\Gamma_{1}:\left(C_{\#}(f, g, \mathbf{S}) \cap \Gamma_{1}\right) \cdot \Gamma_{1}^{\prime}\right]$ coincidence classes, which are either all essential or all inessential. That is, each $\widetilde{\mathbf{S}} \in \mathscr{E}(\tilde{f}, \tilde{g})$ is covered by at most $\left[\Gamma_{1}: \Gamma_{1}^{\prime}\right]$ essential classes. If $C_{\#}(f, g, \mathbf{S}) \cap \Gamma_{1}=C_{\#}(f, g, \mathbf{S}) \cap$ $\Gamma_{1}^{\prime}$ then there are exactly $\left[\Gamma_{1}: \Gamma_{1}^{\prime}\right]$ classes, and Theorem 5.6 implies that they are all essential. 
Corollary 5.10. Suppose $X_{1}, X_{2}$ are manifolds of the same dimension, and neither is a surface with negative Euler characteristic. If $C_{\#}(f, g, \mathbf{S}) \subset \Gamma_{1}$ for every $\mathbf{S} \in \mathscr{E}(f, g)$ and every $\Gamma_{1} \in \mathscr{C}\left(\pi_{1}\right)$, then $\widetilde{N}$ is independent of $\Gamma_{1}: \widetilde{N}\left(f, g, \Gamma_{1}\right)=N(f, g)$ for every $\Gamma_{1}$.

THEOREM 5.11. 1. If $\Gamma_{2}^{\prime}, \Gamma_{2} \in \mathscr{C}\left(\pi_{2}\right)$ with both $\mathscr{\mathscr { C }}\left(f, g, \Gamma_{2}\right)$ and $\mathscr{\mathscr { C }}\left(f, g, \Gamma_{2}^{\prime}\right)$ nonempty and $\Gamma_{2}^{\prime} \subset \Gamma_{2}$, then $\widetilde{L}\left(f, g, \Gamma_{2}\right) \leq$ $\widetilde{L}\left(f, g, \Gamma_{2}^{\prime}\right)$.

2. Let $\rho: \Phi_{2}^{\prime} \rightarrow \Phi_{2}$ be the natural projection, and let $(\tilde{f}, \tilde{g})$ and $\left(\tilde{f}^{\prime}, \tilde{g}^{\prime}\right)$ be the lifts of $(f, g)$ corresponding to $\Gamma_{2}$ and $\Gamma_{2}^{\prime}$ respectively. Then $\widetilde{L}\left(f, g, \Gamma_{2}\right)=\widetilde{L}\left(f, g, \Gamma_{2}^{\prime}\right)$ if and only if, for every $\beta \in \Phi_{2}$, all lifts $\left(\beta^{\prime} \circ \tilde{f}^{\prime}, \tilde{g}\right), \beta^{\prime} \in \rho^{-1}(\beta)$, have $L\left(\beta^{\prime} \circ \tilde{f}^{\prime}, \tilde{g}\right)$ with the same sign.

Proof. Both statements follow immediately from the equality

$$
L(\beta \circ \tilde{f}, \tilde{g})=\sum_{\beta^{\prime} \in \rho^{-1}(\beta)} L\left(\beta^{\prime} \circ \tilde{f}^{\prime}, \tilde{g}^{\prime}\right) .
$$

6. Infrasolvmanifolds. The goal of this work is to use the machinery developed in the previous section to extend the results of [18] and [19] to infrasolvmanifolds. In this section, we briefly review the topology of infrasolvmanifolds, before turning to coincidence theory on infrasolvmanifolds in the next section. Three subclasses of infrasolvmanifolds will merit special attention: nilmanifolds, infranilmanifolds and solvmanifolds. The topology of nilmanifolds is described in [17]; solvmanifolds in [20] and [24]; infranilmanifolds in [1]; and infrasolvmanifolds in [12].

We begin with the constructive definition of infrasolvmanifolds. Let $S$ be a solvable connected simply connected Lie group, and consider the Lie group $G=S \rtimes \operatorname{Aut}(S)$. $G$ acts on $\mathscr{S}$ by $(s, \alpha) \cdot s^{\prime}=s \alpha\left(s^{\prime}\right)$. If $\pi \subset G$ is a torsion-free subgroup with finite projection $\Phi$ onto $\operatorname{Aut}(S)$, then $M=\pi \backslash S$ is an infrasolvmanifold. If $\Gamma=\pi \cap S$, then $\widetilde{M}=\Gamma \backslash S$ is a covering space of $M$ with covering group $\Phi=\pi / \Gamma . M$ is connected, and is compact if and only if $\pi$ is uniform in $S \rtimes K$, or equivalently if and only if $\Gamma$ is uniform in $S$. We will restrict ourselves to the compact case. This same construction generates the three subclasses of manifolds mentioned: $M$ is an infranilmanifold if $S$ is nilpotent, and a nilmanifold if $S$ is nilpotent and $\Phi=1$. $M$ is a solvmanifold if $\Phi$ is solvable, and a special solvmanifold if $\Phi=1$. Clearly, every infrasolvmanifold has a finite regular cover by 
a solvmanifold (indeed, by a special solvmanifold) and every infranilmanifold has a finite regular cover by a nilmanifold. In all cases, the universal cover $S$ is contractible, so the manifold is aspherical with $\pi_{1}(M) \cong \pi$.

Compact infrasolvmanifolds are determined up to homeomorphism by their fundamental group, which must be torsion-free and finitely generated. Recall that for any property $\mathrm{P}$ of groups, a group $G$ is virtually $\mathrm{P}$ if there is a normal subgroup of finite index $H$ which has property $\mathrm{P}$. Similarly, a group is poly-P if there is a normal series $\left\{G_{i}\right\}$ for $G$ such that each subquotient $G_{i} / G_{i+1}$ has property P. In particular, $G$ is polycyclic if there is a normal series with $G_{i} / G_{i+1} \cong$ Z. Another group-theoretic definition: A strongly torsion-free $\mathscr{S}$ group is a group $\pi$ with a finitely generated torsion-free nilpotent $\Gamma \triangleleft \pi$ such that $\pi / \Gamma$ is free abelian.

THEOREM 6.1. There is a one-to-one correspondence between homeomorphism classes of nilmanifolds, infranilmanifolds, solvmanifolds and infrasolvmanifolds and isomorphism classes of the following categories of torsion-free finitely generated groups: nilpotent groups, virtually nilpotent groups, strongly torsion-free solvable groups and virtually polycyclic groups.

If $\pi$ is the fundamental group of $M$, then $\pi$ is virtually polycyclic, with $\operatorname{dim}(M)=r k(\pi)=n$, where $n$ is the number of infinite cyclic summands in the "virtual" polycyclic decomposition of $\pi$.

In general, infranilmanifolds, solvmanifolds and infrasolvmanifolds are not orientable as manifolds. The Klein bottle, for example, is both an infranilmanifold and a solvmanifold. However, all nilmanifolds are orientable, and of course, if $M$ is a non-orientable infrasolvmanifold, there is an orientable infrasolvmanifold covering it, which is a solvmanifold or infranilmanifold if $M$ is. It is well known that all solvmanifolds have zero Euler characteristic; hence all infrasolvmanifolds have $\chi(M)=0$. Finally, all pairs of infrasolvmanifolds of the same dimension are Wenken pairs. For dimensions 3 or more, this follows from the result mentioned in $\S 2$. In dimensions 1 and 2 , the requirement $\chi(M)=0$ limits the possibilities to the circle, torus and Klein bottle. All of these are in fact solvmanifolds, for which the result is established in (among other places) [19].

7. Coincidence numbers on infrasolvmanifolds. The tools we will use to study coincidence numbers for infrasolvmanifolds are the machinery developed in $\S 5$ and the following results: 
THEOREM 7.1 [19, Thm. 1]. If $M_{1}, M_{2}$ are compact connected orientable solvmanifolds of the same dimension, and $\mathbf{S} \in \mathscr{R}(f, g)$, then $\operatorname{Ind}(f, g, \mathbf{S}) \in\{-1,0,1\}$.

THEOREM 7.2 [19, Thm. 2]. If $M_{1}, M_{2}$ are compact connected orientable solvmanifolds of the same dimension, then $N(f, g) \geq|L(f, g)|$ for every $f, g: M_{1} \rightarrow M_{2}$. Moreover, if $M_{2}$ is a nilmanifold, then $N(f, g)=|L(f, g)|$ for every $(f, g)$.

It is now a simple matter to combine these and obtain our main results.

THEOREM 7.3. If $M_{1}$ and $M_{2}$ are compact connected infrasolvmanifolds of the same dimension, and $\pi_{1} \in \mathscr{H}\left(f, g, \pi_{2}\right)$, then $\operatorname{Ind}(f, g, \mathbf{S}) \in\{-1,0,1\}$ for every $\mathbf{S} \in \mathscr{R}(f, g)$.

Proof. Choose a solvable $\Gamma_{2}^{\prime} \in \mathscr{C O}\left(\pi_{2}\right)$ and a solvable $\Gamma_{1}^{\prime} \in$ $\mathscr{C}\left(f, g, \Gamma_{2}^{\prime}\right)$. In the $\Gamma_{1}^{\prime}-\Gamma_{2}^{\prime}$ lifting diagram, $\widetilde{M}_{1}$ and $\widetilde{M}_{2}$ are orientable solvmanifolds, and by Theorem 7.1, any coincidence class $\widetilde{\mathbf{S}}$ covering $\mathbf{S}$ has $\operatorname{Ind}(\tilde{f}, \tilde{g}, \widetilde{\mathbf{S}}) \in\{-1,0,1\}$. But

$$
\left|\Phi_{1}\right| \operatorname{Ind}(f, g, \mathbf{S})=\sum_{\widetilde{\mathbf{S}} \in \mathscr{S}} \operatorname{Ind}(\beta \circ \tilde{f}, \tilde{g}),
$$

so

$$
\begin{aligned}
\left|\Phi_{1}\right||\operatorname{Ind}(f, g, \mathbf{S})| & \leq \sum_{\widetilde{\mathbf{S}} \in \mathscr{S}}|\operatorname{Ind}(\beta \circ \tilde{f}, \tilde{g})| \\
& \leq|\mathscr{S}|=\left[\pi_{1}: C_{\#}(f, g, \mathbf{S}) \Gamma_{1}\right] .
\end{aligned}
$$

But $\mathbf{S}$ is covered by at most $|\Phi|$ coincidence classes, so

$$
\left|\Phi_{1}\right||\operatorname{Ind}(f, g, \mathbf{S})| \leq|\Phi| \quad \text { and }|\operatorname{Ind}(f, g, \mathbf{S})| \leq 1 .
$$

THEOREM 7.4. If $M_{1}$ and $M_{2}$ are compact connected infrasolvmanifolds of the same dimension, then $\widetilde{N}\left(f, g, \Gamma_{1}\right) \geq \widetilde{L}\left(f, g, \Gamma_{2}\right)$ for every $f, g: M_{1} \rightarrow M_{2}$ and every $\Gamma_{2} \in \mathscr{C}\left(\pi_{2}\right), \Gamma_{1} \in \mathscr{I} \mathscr{C}\left(f, g, \Gamma_{2}\right)$. In particular, $N(f, g) \geq \max \left\{\widetilde{L}\left(f, g, \Gamma_{2}\right) \mid \mathscr{H}\left(f, g, \Gamma_{2}\right) \neq \varnothing\right\}$.

Proof. Choose a solvable $\Gamma_{2}^{\prime} \in \mathscr{C} \mathscr{O}\left(\pi_{2}\right)$ and a solvable $\Gamma_{1}^{\prime} \in$ $\mathscr{C}\left(f, g, \Gamma_{2}^{\prime}\right)$. In the $\Gamma_{1}^{\prime}-\Gamma_{2}^{\prime}$ lifting diagram, $\widetilde{M}_{1}$ and $\widetilde{M}_{2}$ are orientable solvmanifolds, and by Theorem 7.2, $N(\beta \circ \tilde{f}, \tilde{g}) \geq$ $|L(\beta \circ \tilde{f}, \tilde{g})|$. Then for any such choice of $\Gamma_{2}^{\prime}$ and $\Gamma_{1}^{\prime}, \tilde{N}\left(f, g, \Gamma_{1}^{\prime}\right)$ $\geq \widetilde{L}\left(f, g, \Gamma_{2}^{\prime}\right)$. But for any $\Gamma_{1}, \Gamma_{2}$ with $\Gamma_{1} \in \mathscr{C} \mathscr{C}\left(f, g, \Gamma_{2}\right)$, there 
exists a solvable $\Gamma_{2}^{\prime} \in \mathscr{C} \mathscr{O}\left(\pi_{2}\right)$ and a solvable $\Gamma_{1}^{\prime} \in \mathscr{\mathscr { C }}\left(f, g, \Gamma_{2}^{\prime}\right)$ with $\Gamma_{i}^{\prime} \subseteq \Gamma_{i}$. Then $\tilde{N}\left(f, g, \Gamma_{1}\right) \geq \widetilde{N}\left(f, g, \Gamma_{1}^{\prime}\right) \geq \widetilde{L}\left(f, g, \Gamma_{2}^{\prime}\right) \geq$ $L\left(f, g, \Gamma_{2}\right)$.

COROLlary 7.5. If $M$ is a compact connected infrasolvmanifold, then $N(f) \geq|L(f)|$ for every $f: M \rightarrow M$.

Can the numbers $\widetilde{L}\left(f, g, \Gamma_{2}\right)$ ever compute $N(f, g)$ ? That is, is there a systematic choice of $\Gamma_{2} \in \mathscr{C}\left(\pi_{2}\right)$ with $\widetilde{L}\left(f, g, \Gamma_{2}\right)=N(f, g)$ for all $(f, g)$ ? The natural route to such an equality is to find $\Gamma_{1} \in$ $\mathscr{C}\left(f, g, \Gamma_{2}\right)$ with $N(f, g)=\widetilde{N}\left(f, g, \Gamma_{1}\right)=\widetilde{L}\left(f, g, \Gamma_{2}\right)$. If $\Gamma_{2}$ is nilpotent, the second equality will hold [19, Thm. 2]. To obtain the first, we need $C_{\#}(f, g, \mathbf{S}) \subseteq \Gamma_{1}$ for all essential coincidence classes $\mathbf{S}$. The following results give sufficient conditions for this.

COROLlaRY 7.6. If $M_{1}$ and $M_{2}$ are compact connected infrasolvmanifolds of the same dimension, and $\pi_{1} \in \mathscr{H}\left(f, g, \pi_{2}\right)$, then $C_{\#}(f, g, \mathbf{S}) \subset \Gamma_{1}$ for every $\Gamma_{1} \in \mathscr{C}\left(\pi_{1}\right)$ and every essential coincidence class $\mathbf{S} \in \mathscr{E}(f, g)$.

Proof. If $\mathbf{S}$ is essential, then from Theorem 7.3, $\operatorname{Ind}(f, g, \mathbf{S})=$ \pm 1 , and $\left|\Phi_{1}\right|=\left[\pi_{1}: C_{\#}(f, g, \mathbf{S}) \Gamma_{1}\right]$. That is, $C_{\#}(f, g, \mathbf{S}) \subset \Gamma_{1}$.

LemMa 7.7 [19, Thm. 3.4]. If $M_{1}$ and $M_{2}$ are compact connected solvmanifolds of the same dimension, then for every $f, g: M_{1} \rightarrow M_{2}$, $C_{\#}(f, g, \mathbf{S})=1$ for every $\mathbf{S} \in \mathscr{E}(f, g)$.

Lemma 7.8. Suppose $M_{1}, M_{2}$ are infrasolvmanifolds of the same dimension and $M_{2}$ is a nilmanifold (resp. solvmanifold, infranilmanifold). Then either $M_{1}$ is a nilmanifold (resp. solvmanifold, infranilmanifold), or $N(f, g)=0$ for all $f, g: M_{1} \rightarrow M_{2}$.

Proof. We will prove the case of $M_{2}$ an infranilmanifold, $M_{1}$ not an infranilmanifold. Since $M_{2}$ is an infranilmanifold, there is a nilpotent $\Gamma_{2} \in \mathscr{C}\left(\pi_{2}\right)$. The derived series $\Gamma_{2}^{k}=\left[\Gamma_{2}, \Gamma_{2}^{k-1}\right]$ descends to 1 : there is an $n$ with $\Gamma_{2}^{n}=1$. Take $\Gamma_{1} \in \mathscr{C}\left(f, g, \Gamma_{2}\right)$. Since $M_{1}$ is not an infranilmanifold, $\Gamma_{1}$ is not nilpotent, and $\Gamma_{1}^{n} \neq 1$. But $f_{\#}, g_{\#}\left(\Gamma_{1}\right) \subseteq \Gamma_{2}$, so $f_{\#}, g_{\#}\left(\Gamma_{1}^{n}\right) \subseteq \Gamma_{2}^{n}=1$. Then $K=\operatorname{ker}\left(f_{\#}\right) \cap \operatorname{ker}\left(g_{\#}\right)$ has $\operatorname{rk}(K)>1$ and $Q=\pi_{1} / K$ is a finitely generated torsion-free virtually poly- $\mathbf{Z}$ group. Of course, if $\rho: \pi_{1} \rightarrow Q$ is the natural projection, there are homomorphisms $\phi, \gamma: Q \rightarrow \pi_{2}$ with $f_{\#}=\phi \circ \rho$ and 
$g_{\#}=\gamma \circ \rho$. From [2], there is then a compact connected infrasolvmanifold $\bar{M}$ with $\pi_{1}(\bar{M})=Q$ and $\operatorname{dim}(\bar{M})=\operatorname{rk}(Q)<\operatorname{rk}\left(\pi_{1}\right)=$ $\operatorname{dim}\left(M_{1}\right)=\operatorname{dim}\left(M_{2}\right)$. Moreover, since $M_{1}, M_{2}$ and $\bar{M}$ are all aspherical, the fundamental group diagram

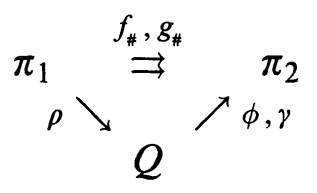

can be realized as the diagram of maps

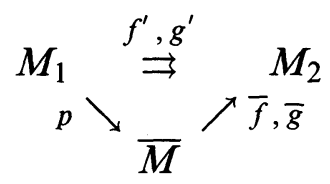

with $f^{\prime} \simeq f$ and $g^{\prime} \simeq g$. Moreover, $\bar{f}$ and $\bar{g}$ may be assumed to be smooth.

Now consider the map $\bar{M} \stackrel{\bar{f} \times \bar{g}}{\rightarrow} M_{2} \times M_{2}$. By transversality, $\bar{f} \times \bar{g}$ is homotopic to a map $\bar{f}^{\prime} \times \bar{g}^{\prime}$ which is transverse to the diagonal $\Delta\left(M_{2}\right)$. Since $\operatorname{dim}(\bar{M})+\operatorname{dim}\left(\Delta\left(M_{2}\right)\right)<\operatorname{dim}\left(M_{2} \times M_{2}\right)$, this really means that $\bar{f}^{\prime} \times \bar{g}^{\prime}$ misses the diagonal, or that $\operatorname{Coin}\left(\bar{f}^{\prime}, \bar{g}^{\prime}\right)=\varnothing$. Then the maps $f^{\prime \prime}, g^{\prime \prime}: M_{1} \rightarrow M_{2}$ given by $f^{\prime \prime}=\bar{f}^{\prime} \circ p, g^{\prime \prime}=\bar{g}^{\prime} \circ p$ are homotopic to $f$ and $g$ respectively, and have $\operatorname{Coin}\left(f^{\prime \prime}, g^{\prime \prime}\right)=\varnothing$.

THEOREM 7.9. Suppose $M_{1}$ an $n$-dimensional infrasolvmanifold and either $M_{2}$ is an n-dimensional infranilmanifold and solvmanifold, or $\pi_{1} \in \mathscr{C}\left(f, g, \pi_{2}\right)$. Then $N(f, g)=\widetilde{L}\left(f, g, \Gamma_{2}\right)$ for every nilpotent $\Gamma_{2} \in \mathscr{C}\left(\pi_{2}\right)$.

Proof. First, suppose $M_{2}$ is a solvmanifold. If $M_{1}$ is not an infranilmanifold, or is not a solvmanifold, Lemma 7.8 implies that $N(f, g)=0$ for all $(f, g)$. Then for all $\Gamma_{2}, \widetilde{L}\left(f, g, \Gamma_{2}\right)=0$ as well. We can therefore assume that $M_{1}$ is likewise both an infranilmanifold and a solvmanifold. Since it is a solvmanifold, Corollary 5.10 implies $N(f, g)=\widetilde{N}\left(f, g, \Gamma_{1}\right)$ for all $\Gamma_{1}$. On the other hand, since it is an infranilmanifold, $\widetilde{N}\left(f, g, \Gamma_{1}\right) \geq \widetilde{L}\left(f, g, \Gamma_{2}\right)$ for every nilpotent $\Gamma_{2} \in \mathscr{C}\left(\pi_{2}\right)$ and $\Gamma_{1} \in \mathscr{H}\left(f, g, \Gamma_{2}\right)$. The argument for the case $\pi_{1} \in \mathscr{C}\left(f, g, \pi_{2}\right)$ is the same, only substituting Corollary 7.6 for Lemma 7.7.

COROllary 7.10. If $M$ is an infranilmanifold, then $N(f)=$ $\widetilde{L}\left(f\right.$, id, $\left.\Gamma_{2}\right)$ for every nilpotent $\Gamma_{2} \in \mathscr{C}\left(\pi_{2}\right)$ and every $f: M \rightarrow M$. 
The Klein bottle $K$ is perhaps the best-known example of a space which is both a solvmanifold and an infranilmanifold, but not a nilmanifold. The fundamental group $\pi_{1}(K) \cong \mathbf{Z} \rtimes \mathbf{Z}$, where $n \in \mathbf{Z}$ acts on $m \in \mathbf{Z}$ by $n \cdot m=(-1)^{n} m$. The orientation subgroup $O(\pi)=2 \mathbf{Z} \rtimes \mathbf{Z} \cong \mathbf{Z}^{2}$ is invariant under all homomorphisms, so $O(\pi) \in \mathscr{C}(f, g, O(\pi))$ for all $(f, g)$. If $\alpha$ is the generator of $\pi / O(\pi)$ and $(\tilde{f}, \tilde{g})$ are lifts of $(f, g)$ in the $O(\pi)-O(\pi)$ lifting diagram, then $N(f, g)=|L(\tilde{f}, \tilde{g})|+|L(\alpha \circ \tilde{f}, \tilde{g})|$. In particular, $N(f)=|L(\tilde{f})|+|L(\alpha \circ \tilde{f})|$.

8. The Jiang condition. As was mentioned in $\S 1$, the Jiang subgroup and Jiang condition [15] provide additional tools for estimating and calculating Nielsen numbers. In this section, we consider how the results described in this paper relate to the Jiang condition. Given a map $f: X \rightarrow X$ and $x \in \operatorname{Fix}(f)$, define

$$
J(f)=\left\{\omega \in \pi=\pi_{1}(X, x) \mid \omega=[H(x,-)] \text { for some } H: f \simeq f\right\} .
$$

Some of the important properties of $J(f)$ are:

1. $J(f)$ is independent of the base point chosen.

2. $J(f) \subseteq \mathbf{C}_{\pi}\left(f_{\#}(\pi)\right)$, the centralizer of $f_{\#}(\pi)$ in $\pi$, with equality if $X$ is aspherical.

3. $J(X)=J($ id $) \subseteq J(f)$ for all $f$.

4. If $f_{\#}(\pi) \subseteq J(f)$, then all fixed point classes have the same index.

In particular, in our setting of an aspherical manifold, if $f_{\#}(\pi)$ is abelian, then $L(f)=N(f) \cdot \operatorname{Ind}(f, \mathbf{S})$ for any fixed point class $\mathbf{S}$, with $N(f)=0$ if $\operatorname{Ind}(f, \mathbf{S})=0$. Of course, $f_{\#}(\pi)$ will be abelian for all $f$ if and only if $\pi$ is abelian. Thus the only infrasolvmanifolds for which the Jiang condition is satisfied for all maps are tori. Thus the Jiang condition does not contribute to our goal of finding conditions on an aspherical manifold which allow $N(f)$ to be estimated or computed for all self-maps. However, for some maps on infrasolvmanifolds, it does allow us to sharpen the inequality $|L(f)| \leq N(f)$ to equality. Namely, we have:

THEOREM 8.1. If $f: S \rightarrow S$ is a self map of an infrasolvmanifold with $f_{\#}(\pi)$ abelian, then $N(f)=|L(f)|$.

9. Conclusion. Two directions for further study naturally suggest themselves: refining these results in the infrasolvmanifold category, and extending the results to larger categories of manifolds. To refine these results, we need to know two things: for which spaces is 
$\tilde{N}\left(f, g, \Gamma_{1}\right)$ independent of $\Gamma_{1}$ for all $f$ and $g$ ? For which spaces is $N(f, g)=|L(f, g)|$ for all $f$ and $g$ ? Of course, the first question hinges on the behavior of $C_{\#}(f, g, \mathbf{S})$ for essential classes $\mathbf{S}$. It seems likely that $C_{\#}(f, g, \mathbf{S})=1$ for essential classes on all infranilmanifolds; the situation is less clear for infrasolvmanifolds. As to the question of equality of the Nielsen number and Lefschetz number, the natural conjecture is that equality occurs for all $f$ and $g$ if and only if the image manifold is a nilmanifold.

To extend the results to larger classes of manifolds, consider infrasolvmanifolds as spherical manifolds with torsion-free virtually polycyclic fundamental groups. There are several natural extensions of this class of groups: torsion-free elementary groups; torsion-free amenable groups, torsion-free "no-free groups" (i.e. groups which do not contain $F_{2}$, cf. [21]). For each, there is a corresponding category of compact aspherical manifolds. To what extent does the Lefschetz number estimate or compute the Nielsen number in these larger categories? In particular, is it true in any of these larger categories that $N(f) \geq|L(f)|$ for all self-maps $f$ ?

\section{REFERENCES}

[1] L. Auslander, Bieberbach's theorems on space groups and discrete uniform subgroups of Lie groups, Ann. of Math., 71 (1960), 579-590.

[2] L. Auslander and F. E. A. Johnson, On a conjecture of C. T. C. Wall, J. London Math. Soc., (2) 14 (1976), 331-332.

[3] D. V. Anosov, The Nielsen number of maps of nil-manifolds, Russian Math. Surveys, 40 (1985), 149-150.

[4] R. Brooks, Coincidences, roots and fixed points, Ph.D. dissertation, University of California-Los Angeles, 1967.

[5] - On removing coincidences of two maps when only one, rather than both, of them may be deformed by a homotopy, Pacific J. Math., 39 (1971), 45-52.

[6] - On the sharpness of the $\Delta_{2}$ and $\Delta_{1}$ Nielsen numbers, J. Reine Angew. Math., 259 (1973), 101-108.

[7] R. Brooks and R. Brown, A lower bound for the $\Delta$-Nielsen number, Trans. Amer. Math. Soc., 143 (1969), 555-564.

[8] R. Brooks, R. Brown, J. Pak and D. Taylor, Nielsen numbers of maps of tori, Proc. Amer. Math. Soc., 52 (1975), 398-400.

[9] R. Brooks and R. Wong, On changing fixed points and coincidences to roots, to appear in Proc. Amer. Math. Soc.

[10] E. Fadell, On a coincidence theorem of F. B. Fuller, Pacific J. Math., 15 (1965), 825-834.

[11] E. Fadell and S. Husseini, On a theorem of Anosov on Nielsen numbers for nilmanifolds, Nonlinear Functional Analysis and its Applications (Maratea, 1985), 47-53 NATO Adv. Sci. Inst. Ser. C: Math. Phys. Sci., 173, Reidel, DordrechtBoston, Mass., 1986. 
[12] F. T. Farrell and L. E. Jones, Classical Aspherical Manifolds, CBMS Lecture Notes, \# 75, Amer. Math. Soc., Providence, RI 1990.

[13] F. B. Fuller, The Homotopy Theory of Coincidences, Ph.D. thesis, Princeton University, 1951.

[14] J. Jezierski, The Nielsen number product formula for coincidences, Fund. Math., 134 (1989), 183-212.

[15] B. Jiang, Lectures on Nielsen fixed point theory, Contemporary Mathematics, vol. 14, Amer. Math. Soc., Providence, RI 1983.

[16] S. Kwasik and K. B. Lee, The Nielsen numbers of homotopically periodic maps of infranilmanifolds, J. London Math. Soc., (2) 38 (1988), 544-554.

[17] A. Mal'cev, On a class of homogeneous spaces, Amer. Math. Soc. Transl. (2), vol. 39, Amer. Math. Soc., Providence, RI, 1951, pp. 276-307.

[18] C. McCord, Nielsen numbers and Lefschetz numbers on solvmanifolds, Pacific J. Math., 147 (1991), 153-164.

[19] _ Lefschetz and Nielsen coincidence numbers on nilmanifolds and solvmanifolds, to appear in Topology Appl.

[20] G. Mostow, Factor spaces of solvable groups, Ann. of Math., 60 (1954), 1-27.

[21] A. Paterson, Amenability, Mathematical Surveys and Monographs, vol. 20, Amer. Math. Soc., Providence, RI, 1988.

[22] H. Schirmer, Mindestzahlen von Koinzidenpunkten, J. Reine Angew. Math., 194 (1955), 21-39.

[23] J. Vick, Homology Theory, Academic Press, New York 1973.

[24] H.-C. Wang, Discrete subgroups of solvable Lie groups I, Ann. of Math., 64 (1956), 1-19.

Received February 26, 1991 and in revised form June 6, 1991. Supported in part by DARPA Applied and Computational Mathematics Program and by the Charles Phelps Taft Memorial Fund.

INSTITUTE FOR DYNAMICS

UNIVERSITY OF CINCINNATI

Cincinnati, OH 45221-0025 


\section{PACIFIC JOURNAL OF MATHEMATICS EDITORS}

\author{
V. S. VARADARAJAN \\ (Managing Editor) \\ University of California \\ Los Angeles, CA 90024-1555 \\ Herbert Clemens \\ University of Utah \\ Salt Lake City, UT 84112 \\ F. Michael Christ \\ University of California \\ Los Angeles, CA 90024-1555 \\ THOMAS ENRIGHT \\ University of California, San Diego \\ La Jolla, CA 92093
}

\author{
Nicholas ERcolani \\ University of Arizona \\ Tucson, AZ 85721 \\ R. FINN \\ Stanford University \\ Stanford, CA 94305 \\ VAughan F. R. JONES \\ University of California \\ Berkeley, CA 94720 \\ C. C. Moore \\ University of California \\ Berkeley, CA 94720 \\ MARTIN ScharlemanN \\ University of California \\ Santa Barbara, CA 93106 \\ Harold Stark \\ University of California, San Diego \\ La Jolla, CA 92093
}

STEVEN KeRCKHOFF

Stanford University

Stanford, CA 94305

\begin{tabular}{|c|c|c|c|c|}
\hline \multicolumn{5}{|c|}{ ASSOCIATE EDITORS } \\
\hline R. Arens & $\begin{array}{ll}\text { E. F. BECKENBACH } & \text { B. H } \\
(1906-1982) & \end{array}$ & NeUmanN & $\begin{array}{c}\text { F. WoLF } \\
(1904-1989)\end{array}$ & K. Yoshida \\
\hline \multicolumn{5}{|c|}{ SUPPORTING INSTITUTIONS } \\
\hline \multicolumn{2}{|c|}{ UNIVERSITY OF ARIZONA } & \multicolumn{3}{|c|}{ UNIVERSITY OF OREGON } \\
\hline \multicolumn{2}{|c|}{ UNIVERSITY OF BRITISH COLUMBIA } & \multicolumn{3}{|c|}{ UNIVERSITY OF SOUTHERN CALIFORNIA } \\
\hline \multicolumn{2}{|c|}{ CALIFORNIA INSTITUTE OF TECHNOLOGY } & \multicolumn{3}{|c|}{ STANFORD UNIVERSITY } \\
\hline \multicolumn{2}{|c|}{ UNIVERSITY OF CALIFORNIA } & \multicolumn{3}{|c|}{ UNIVERSITY OF HAWAII } \\
\hline \multicolumn{2}{|c|}{ MONTANA STATE UNIVERSITY } & \multicolumn{3}{|c|}{ UNIVERSITY OF TOKYO } \\
\hline \multicolumn{2}{|c|}{ UNIVERSITY OF NEVADA, RENO } & \multicolumn{3}{|c|}{ UNIVERSITY OF UTAH } \\
\hline & \multicolumn{3}{|c|}{ WASHINGTON STATE UNIVERSITY } \\
\hline \multicolumn{2}{|c|}{ OREGON STATE UNIVERSITY } & \multicolumn{3}{|c|}{ UNIVERSITY OF WASHINGTON } \\
\hline
\end{tabular}

The Supporting Institutions listed above contribute to the cost of publication of this Journal, but they are not owners or publishers and have no responsibility for its content or policies.

Mathematical papers intended for publication in the Pacific Journal of Mathematics should be in typed
form or offset-reproduced (not dittoed), double spaced with large margins. Please do not use built up fractions
in the text of the manuscript. However, you may use them in the displayed equations. Underline Greek letters
in red, German in green, and script in blue. The first paragraph must be capable of being used separately as
a synopsis of the entire paper. In particular it should contain no bibliographic references. Please propose a
heading for the odd numbered pages of less than 35 characters. Manuscripts, in triplicate, may be sent to any
one of the editors. Please classify according to the 1991 Mathematics Subject Classification scheme which
can be found in the December index volumes of Mathematical Reviews. Supply name and address of author
to whom proofs should be sent. All other communications should be addressed to the managing editor, or
Elaine Barth, University of California, Los Angeles, California $90024-1555-05$.
There are page-charges associated with articles appearing in the Pacific Journal of Mathematics. These
charges are expected to be paid by the author's University, Government Agency or Company. If the author or
authors do not have access to such Institutional support these charges are waived. Single authors will receive
50 free reprints; joint authors will receive a total of 100 free reprints. Additional copies may be obtained at
cost in multiples of 50 .

The Pacific Journal of Mathematics (ISSN 0030-8730) is published monthly except for July and August. Regular subscription rate: $\$ 190.00$ a year (10 issues). Special rate: $\$ 95.00$ a year to individual members of supporting institutions.

Subscriptions, orders for numbers issued in the last three calendar years, and changes of address should be sent to Pacific Journal of Mathematics, P.O. Box 969, Carmel Valley, CA 93924, U.S.A. Old back numbers obtainable from Kraus Periodicals Co., Route 100, Millwood, NY 10546.

The Pacific Journal of Mathematics at P.O. Box 969, Carmel Valley, CA 93924 (ISSN 0030-8730) is published monthly except for July and August. Second-class postage paid at Carmel Valley, California 93924, and additional mailing offices. Postmaster: send address changes to Pacific Journal of Mathematics, P.O. Box 969, Carmel Valley, CA 93924.

PUBLISHED BY PACIFIC JOURNAL OF MATHEMATICS, A NON-PROFIT CORPORATION Copyright (C) 1992 by Pacific Journal of Mathematics 


\section{Pacific Journal of Mathematics}

\section{Vol. 154, No. $2 \quad$ June, 1992}

Manuel (Rodriguez) de León, J. A. Oubiña, P. R. Rodrigues and

Modesto R. Salgado, Almost $s$-tangent manifolds of higher order . . . . 201

Martin Engman, New spectral characterization theorems for $S^{2} \ldots \ldots \ldots 215$

Yuval Zvi Flicker, The adjoint representation $L$-function for GL $(n) \ldots \ldots 231$

Enrique Alberto Gonzalez-Velasco and Lee Kenneth Jones, On the range

of an unbounded partly atomic vector-valued measure . ............ 245

Takayuki Hibi, Face number inequalities for matroid complexes and

Cohen-Macaulay types of Stanley-Reisner rings of distributive

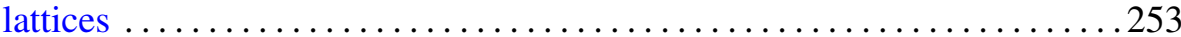

Hervé Jacquet and Stephen James Rallis, Kloosterman integrals for skew

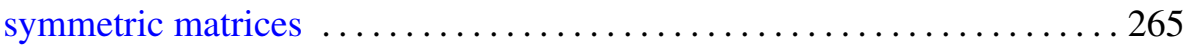

Shulim Kaliman, Two remarks on polynomials in two variables .........285

Kirk Lancaster, Qualitative behavior of solutions of elliptic free boundary problems ..........................................297

Feng Luo, Actions of finite groups on knot complements . ........... 317

James Joseph Madden and Charles Madison Stanton, One-dimensional

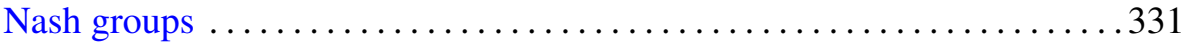

Christopher K. McCord, Estimating Nielsen numbers on

infrasolvmanifolds ......................................... 345

Gordan Savin, On the tensor product of theta representations of $\mathrm{GL}_{3}$ 369

Gerold Wagner, On means of distances on the surface of a sphere. II.

(Upper bounds) 